IV.

\title{
Jahresbericht über die Tätigkeit der Ohrenklinik des Kommunehospitals zu Kopenhagen im Jahre 1909.
}

(Direktor: Prof. Dr. Holger Mygind).

Yon

Dr. P. Tetens Hald,

Privatdozent und 1. Assistenzarzt der Klinik.

In der Poliklinik wurden während des Jahres 19091476 Patienten behandelt. Die Zahl der Konsultationen betrug: 12119.

In der stationären Klinik war der Krankenbestand am 1. Januar 1909 4 Männer, 2 Frauen und 12 Kinder. Neu aufgenommen wurden 322 Kranke, und zwar 99 Männer, 61 Frauen und 162 Kinder. Entlassen wurden 95 Männer, 56 Frauen und 151 Kinder, im ganzen 302 , gestorben sind 6 Männer, 3 Frauen und 14 Kinder, also 23, demnach eine Mortalität von 7,1 Proz. Der Krankenbestand am 1. Januar 1900 betrug 2 Männer, 4 Frauen und 9 Kinder.

Als Assistenten fungierten Dr. P. Tetens Hald, Dr. N. Rh. Blegvad, Dr. C. Speyer (bis 28. Febr. 1909) und Dr. F. Ko c h (vom 1. März 1909). Infolge der im vorigen Jahresbericht erwähnten Erbøhung der Bettanzahl auf 33 (für Ohren- und Augenklinik), ist es möglich gewesen, nicht nur mehr Patienten aufzunehmen, sondern auch dieselben in der Klinik länger verbleiben zu lassen, was bei den oft sehr ernsten Krankheiten sehr erwinscht war.

Übersicht äber die wiehtigsten im Jahre 1909 in der Station behandelten Ohren- und Nasenkrankbeiten

Abscessus cerebri

$"$ colli

" epiduralis

, perisinuosus

Männer Frauen

" pulmon.

" subduralis

" subperiost.reg. mastoid. 3

Cancer nasi

Commot. aur, int. Seq.

Cystis cerebri

Encephalitis localis

progre
Epiphlebitis sin. transv. et sigmoid. 2

1.1

1

Fibroma rhinopharyngis

Fistula capsul. aur. int.

retroauric.

Invasio bacterial. liq. cerebrosp.

Leptomeningit. cerebrosp. epidemica

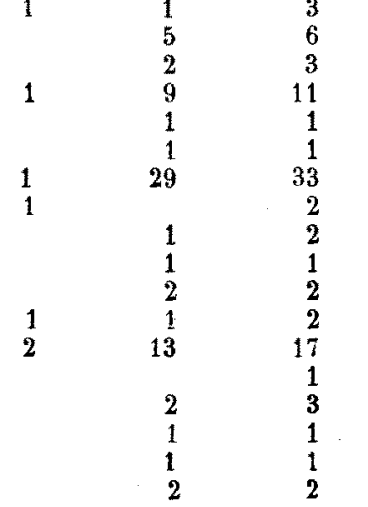


Leptomeningit. purul. diffusa 3 serosa?

tuberculosa

Periostitis acuta reg. mastoid. 4

Mb. Ménière

Ostit. capsul. labyrinthi acuta 1

" proc. mast. acuta 11

" " " " in chron. 4

Otit. ext. diffusa chron.

" "furunc.

3

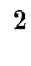

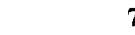

12

" interna acuta diffusa 3

" " chron. circumscr.

" ,,$\quad$ diffusa

" $"$ serosa? 2

" $"$ Seq.

" med. catarrh. acuta 2

" " " chron.

" " suppur. acuta 25

" " " chron. 21

0"osclerosis " " Seq. 1

Papilloma sinus frontalis 1

Paralysis et Paresis $n$. facialis 3

Pachymeningitis externa

Perichondritis auriculae

interna purul.

Prolapsus cerebri

Pyaemia

Pyarthron (otogen.)

Septicaemia

Sinuitis ethmoid. acuta

frontal. chron.
" maxill. acuta
$"$ " chron.
" sphenoidal. acuta

Syphhilis auris" int. chron.

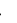

"nasi tertiar. 1

Thrombophlebit. bulb. v. jugul.

Thrombophleb, sinus sigm. pariet. 1

$$
, \quad \nabla \text {. jugul. int. }
$$

Übersicht uber die im Jahre 1909 in der Station wegen Ohren-und Nasenkrankheiten vorgenommenen Operationen.

Ablatio mallei

Conchotomia

Craniotomia

Evulsio polyp. aur.

nasi

Excisio partial. membr. tymp. 29

Excochleatio fistul. retroauric. 1

Incisio cerebelli

$$
\text { proc. mast. } 2
$$

" cerebri

" durae matris

Archiv f. Ohrenheilkunde. Bd. 82
Incisio membr. tymp.

$$
\text { sinus sigmoid. }
$$

Ligatura v. jugul.

Punctio cerebelli

" cerebri

$"$ lumbal.

". sinus sigmoid.

Resect. aur. int.

" " med. tot.

$$
\text { atypic. }
$$


Resect. meato-mastoid.

$"$ proc. mast. ad. mod. Schwartze 53

" " " " atypic. 8

" septi nasi submuc.

sin. ethmoid.

" frontalis

ad mod. Killian 3

Resect. sin. maxill.

4

" " , Caldwell-Luc. 5

" " sigmoid.

9

Sutura primaria post resect.

proc. mast.

Sutura secundaria post resect.

proc. mast.

In 131 Fällen wurde Äthernarkose, in 6 Fällen Chloroform, in 4 Fällen Äther und Chloroform, in 5 Fallen Ätherrausch, in 1 Fall primäre Äthernarkose angewandt, alle ohne Todesfall. In 13 Fällen lokale Anästhesie.

Unserer Gewohnheit gemäß wollen wir auch diesmal nur die unglinstig verlaufenen Fälle ausführlich besprechen. Was die geheilten, mit ernsten Komplikationen verbundenen Fälle betrifft, mögen folgende Angaben genügen. Ein Fall von epiduralem, 6 von perisinuösem Abszeß, 2 von diffuser purulenter Meningitis und 5 von Sinusthrombose wurden durch Operation geheilt.

Übersicht uber die letalen Fälle.

Die Todesursache bietet nur bei drei der Gestorbenen kein otologisohes Interesse dar, indem sie in dem cinen dieser drei Falle Cancer cardiae, im zweiten Cancer oesophagi und im dritten Gastritis phlegmonosa war. Unsere Sterblichkeit ist demnach fur die Ohrenkrankbeiten außerordentlich groß gewesen. Traurig - und lehrreich - ist dabei das mehrfache Vorkommen von postoperativen Todesfällen. Von den Gestorbenen waren sechzehn einer Mastoidoperation unterzogen, was bei 96 Mastoidoperationen eine Mortalität von 16.7 Proz. gibt. Von den seit Tröffnung der stationären Klinik ausgeftihrten 414 Mastoidoperationen sind 39 gestorben, also eine Mortalität von 9.4 Proz.

1. Ander 8 Vigelius H. (No. 38, 1909), 44 Jahre, aufgen. 1. Febr. 1909, gest. 2. Febr. 1909.

Klinische Diagnose: Otit. med. acut, in chron, supp, dext. Neuritis optica. - Abscessus cerebri (cerebelli's) - Tumor cerebri?

Sektionsdiagnose: Otitis media chron. purul. - Meningitis purul. et chron. fibrinosa. - Abscessus cerebri.

Anamnese: Vielleicht OhrfuB als Kind. Laut Mitteilung seiner Frau hat Pat. während der letzten 14 Tagen an Ohrschmerzen und Ohrflub rechts gelitten. Während der letzten 8 Tagen über hämmernde Nackenschmerzen geklagt, die in den zwei letzten Nächten den Schlaf verhindert haben. Vor fünf Tagen einmal Erbrechen (nach Hafergrütze). Kein Fieber oder Schüttelfrost. Als er heute, nachdem er 14 Tage lang das 
Bett gehütet hatte, aufstand, um zum Arzt zu gehen, wurde ibm schwind lig, sonst kein Schwindel. Erst heute nachmittag sel er. unklar geworden, aber während der letzten drei Tage ist er träge gewesen und hat langsam geantwortet. - In der letzten Zeit Obstipation. Wird um $9 \%$ geliefert.

Statuspraesens. Unklar, glaubt bei der Aufnahme schon 24 Stunden in der Klinik gewesen zu sein. Keine Sprachstörungen. Erkennt und benennt gewöhnliche Objekte richtig. Spricht langsam, scheint s low cerebration darzubieten, aber seine leichte Benommenheit macht die Entscheidung schwierig. Keine Paresen. Keine Dysdiadokokinesis oder mangelhafte Antagonistwirkung. Die tiefen Reflexe natürlich. Kein Babinski oder Oppenheim. Kein spontaner Nystagmus. A n d e u tu n v on Kernig beiderseits. Unbodeutende $\mathrm{Nackensteifigkeit.} \mathrm{Temp.} 36.8$. P. 62. A u r dext: Geringe Menge stinkenden Eiters im Gehörgange. Hintergrund von einer ziemlich glatten, roten Fläche gebildet, am wahrscheinlichsten die Labyrinthwand durch einen totalen Trommelfelldefekt gesehen. Proc. mast. dext.: Ohne Infiltration oder Druckschmerz. Kein Druckschmerz am Kranium. A ur. sin. normal Funktionsprobe. G e hö $\mathrm{r}$ rechts für Flïsterstimme ad aurem, für Konversationsstimme $2 \mathrm{~m}$, mit Báránys Lärmapparat im linken $\mathrm{Ohr} b z w .0$ und $1 \mathrm{~m}$. $\mathrm{Kal}$ o $\mathrm{ris} \mathrm{ch}$ e Prüfung ergibt rechts positive Reaktion.

2. Febr. Temp. 36.8.-36.6. Puls 62. Ganz guter Schlaf nach Morphium. Ist etwas unklar gewesen, sowohl gestern abend nach der Untersuchung als früh morgens heute. Bei der Visite völlig klar, mit ungetrübter Erinnerung des gestern Passierten. Antwortet langsam auf alle Fragen nach etwas Nachdenken. Klagt nur noch über Schmerzen im Hinterkopf. Kein Schwindel beim Aufsitzen. Heute kaum Nackensteifigkeit, insbesondere vermag Patient den Kopf in allen Richtungen $z a$ bewegen und zu beugen, ohne daß man eine Spannung der Nackenmuskeln nachweisen kann. Schwache Andeatung von Kernig. $0 \mathrm{ph}$ th a $\mathrm{m} \mathrm{os} \mathrm{k}$ o p is c $\mathrm{h}$ : Beide Papillen geschwollen (ea. :3 Dioptrien). Die Grenzen durch weiBes Exsudat und Hämorrhagien verwischt. Venen stark verbreitert und etwas geschlängelt, Arterien natürlich. Die Veränderungen am stärksten rechts ausgesprochen. Die Papillen gleich, reagieren für Licht und Akkomodation.

Scin Zustand hielt sich den ganzen Tag ziemlich unverändert. Nachmittags war der Puls 42. Von 5 Uhr nachmittags an fing er an heftiger uber Nackenschmerzen za klagen. Um 7 Uhr schrie er auf und wurde unklar, zum Schlnß ganz rasend, wollte aus dem Bette, schlug das Pflegepersonal. Um 9 Uhr trat scheinbar ruhiger Schlaf ein: Gesicht gerötet, die Haut der linken Körperhälfte kühl, rechterseits heiß. Starb plötzlich um $101 / 2$ Uhr nachmittags.

Sektiondes Schläfenbeins und des Gehirns: Völliger Mangel von Trommelfell, Hammer und Amboß. Der Steigbügel sitzt an normaler Stelle. Im Mittelohr eine geringe Menge blutfarbigen Detritus. Das Knochengewebe des Warzenfortsatzes völlig sklerotisch, so daß sích keine Hohlräume außer dem Antrum finden. Antrum kaum erweitert, mit gelblich-grünen Cholesteatom-Nassen gefült. Tegmen antri an seiner zerebralen fiäche grünlich verfärbt; keine makroskopische Perforation. 1)ie Dura ist auf ihrer kranialen Seite; dem verfärbten Tegmen antri entsprechend, mit dicken fleischigen Granulationen besetzt; rings herum starke Injektion der Dura. Die zerebrale Seite der Dura ist an einer ca. $4 \mathrm{~cm}^{2}$ großen Stelle, ca. $4 \mathrm{~cm}$ rückwärts von der Spitze des Temporallappen gelegen, an dessen Unterfläche festgelötet, einer Öffnung in Gehirnrinde entsprechend, welche in eine AbszeBhöhle hineinführt. Die Diameter der Abszebhöhle sind in vertikaler Richtung $2 \mathrm{~cm}$, in frontaler $3 \mathrm{~cm}$, in sagittaler $4 \mathrm{~cm}$. Sie ist mit gelblich-grünem Eiter gefüllt und von einer festeren Kapsel umgeben. An einer umschriebenen Stelle liegt der Abszeß der äußeren Wand des Cornu inferius ganz dicht an, aber man findet weder makroskopische Perforation ins Cornu hinein, noch Eiter im Cornu. Dagegen fanden sich beim Eröffinen des Kraniums die Hïnhäute der Basis des rechten Temporallappens stark eitrig infiltriert. Ferner ein gelatinös eitriger Belag 
an der Unter- und Oberfläche des ganzen Kleinhirns. Die Hirnhäute namentlich an der Basis etwas verdickt, mit zerstreuten narbenartigen Zügen.

Epikrise: In diesem Falle lagen interessante Schwierigkeiten vor, sowohl in diagnostischer als auch in therapeutischer Bezichung.

Was die Diagn os e betrifft, mußte die bei der Einlieferung des Kranken nachgewiesene leichte Trtibung des Sensoriums den Verdacht auf eine intrakranielle Komplikation erwecken. Gegen (akute) Meningitis sprach die beinahe subnormale Temperatur, fur dieselbe der Nackensehmerz, die unbedeutende Naokensteifigkeit und die Andeutung von Kernig. Doch ließen diese letztgenannten Symptome sich wohl auch für die Diagnose einer anderen intrakraniellen Komplikation, Tumor oder besonders AbszeB, verwerten. Da nun slow cerebration vorhanden zu sein schien, und da eine chronische Ohreiterung rechts bestand, mußte man als das wahrscheinlichste einen otogenen Hirnabszeb, und nicht Tumor, diagnostizieren. Die starke Entwickelung der Neuritis optica, dic am nächsten Morgen festgestellt wurde, schien eher für Tumor als Abszel zu sprechen, aber das Vorhandensein der Ohreiterung sehien uns so sehwerwiegend, daß der Pat. bei dem klinischen Unterricht als ein ziemlich sichrer Fall von Hirnabszel vorgeführt wurde. Elie wir aber an die für den folgenden Morgen in Aussieht genommene Operation schreiten könnten, trat nach furibunden Delirien der Tod ein (ca. 26 Stunden nach der Einlieferung). Damit war für die detaillierte Diagnose nicht viel gewonnen. Zwar bestätigte der Vorfall die Annabme einer intrakraniellen Komplikation, aber die mit dem Tode endenden Delirien ließen sich ebensowohl einer plötzlichen Blutung in einen Hirntumor hinein als dem Durchbruch eines Hirnabszesses zuschreiben.

Von einem diagnostischen Filfsmittel, das wir sonst viel verwenden und hoch schätzen, nämlich der Lumbalpunktion, ist in diesem Falle kein Gebrauch gemacht worden. Wenn es so gekommen ist, so ist das einem Zufall zuzuschreiben, indem wir sie in der ohnehin nötigen Narkose ausführen wollten, aber einem Zufall, welchen wir nicht bedauern. Hätten wir nämlich die Lumbalpunktion ausgeführt, und wäre der Fall dann genau ebenso verlaufen, wie er ohne Lumbalpunktion verlief, dann luätten die Gegner der Lumbalpunktion diesen Fall unter den von der Lumbalpunktion verursachten Unglücksfällen auffihren können, und es wäre sehr schwer, ja unmöglich gewesen, die 
Richtigkeit einer solchen Anschauung zu verneinen. Wie es jetzt gegangen ist, illustriert der Fall mit wünschenswertester Deutlichkeit die Tatsache, daß die Lumbalpunktion mitunter auch in solchen Fällen zur Verwendung kommen mag, die vermöge ihrer pathologisch-anatomischen Beschaffenheit auch ohne das durch die Punktion gesetzte Trauma schief verlaufen mässen, und daB es nicht angeht, alle nach einer Lumbalpunktion eintretenden unglücklichen Ereignisse als durch die Punktion versehuldet betrachten zu wollen.

Diese Überlegungen fubren uns zu den von uns erwähnten therapeutischen Schwierigkeiten. In ein er Beziehung ist dieser Ausdruck nicht glücklich gewählt, indem natürlich das therapeutische Vorgehen keinem Zweifel unterliegt, wenn erst die Diagnose Gehirnabszel gestellt worden ist. Aber wir können nicht verhehlen, daß die Möglichkeit, den Kranken durch Operation zu retten, in Fallen wie diesem uns recht gering vorkommt. Der Hirnabszeß hat schon seit langem ein Leiden der Hirnhäute hervorgerufen, das wir durch den Befund chroniseher meningitischer Veränderungen repräsentiert finden. Endlich stirbt der Pat. an einer vom Durehbruch des Abszesses in den basalen Subarachnoidealraum hinein verursachten, in wenigen Stunden tötenden diffusen akuten Leptomeningitis. Was wäre geschehen, falls der Pat. statt ruhig in seinem Bett zn liegen, operiert worden wäre? Es fällt schwer zu glauben, daß selbst das zarteste Vorgehen, Operieren ausschließlich mit der Fraise z. B., nicht den Durchbruch hätte beschleunigen und die Verbreitung der akuten Infektion begünstigen sollen. Es ist, wie anfangs schon gesagt, nieht unsere Meinung, dab man solche Fälle nieht operieren soll. Aber große Befriedigung wird man wohl selten davon haben.

2. Margaretbe Leopold in e C., (No. 134, 1909), 25 Jahre, anfgen. 25. Aug. 1908, entlassen 9. Sept. 1908 . Wieder aufgen. 16. Sept. 1908, entlassen 2. Okt. 1908. Zum dritten Male aufgen. 26. Nov. 1908, gestorben 2. Mai 1909 .

Klin is che Diag n o se: Otit. med. chron. auris sin. - Otit. med. chron. supp, dext. - Osteitis chron. proc. mast. dext. - Vertigo (Mb. Ménière?) - Nach der zweiten Aufnahme: Vertigo (Mb. Ménière?) - Nach der dritten Aufnahme: Abscessus lobi tempor. cerebri dext. - Prolapsus cerebri, - Neuritis optica. - Encephalitis progressiva.

Se $\mathrm{k}$ t i o $\mathrm{s}$ di a g n o se: Meningitis purulenta diffusa hemisph. dext. - Abscessus subduralis dext. - Pachymeningitis circumscripta chron. int. fossae cranii med. dext - Emollitio et Encepbalitis lobi temp. dext. Seq. otit. med. dext.

Anamnese: Seit dem dritten Jabre $O b r f u B$ rechts, mit längeren bis Jahre langen freien Perioden, z. B. kein Ohrfluß rom 13. Jahre bis zu zwei 
Monate vor der Aufnabme. Damals fing das rechte Ohr wieder an zu laufen, auch fanden sich Schmerzen ein. Am 3 . Aug. wurden poliklinisch ein Polyp und eine Menge cholesteatomatöser Massen aus dem rechten Ohr entfernt. Die letzten 4-5 Tage vor der Aufnahme ist ihr etwas schwindlig gewesen, besonders beim Treppenhinabsteigen; sie hat alsdann die Empfindung, als ob die Umgebung sich von links nach rechts drehe. Sie ist nicht gefallen, kein Erbrechen.

Status praesens: Aur. d.: Schleimeiter ohne Geruch im Gehörgang. Große ovale Perf. im hinteren Teil des Trommelfells mit berausdringenden Granulationen nnd cholesteatomatösen Massen. -- A ur. sin.: Katarrhalische Veränderungen des Trommelfells. - Warze nfortsätze: nichts A bnormes. f unktionsprü fung: Ge bör für Flüsterstimme rechts $0.5 \mathrm{~m}$ (mit Báránys Lärmapparat im linken Ohr). Kein spontaner Nytagmus. Nur schwindlig wenn sie sich umdreht. Besteht gut die gewöhnlichen Gleichgewichtsproben. Fistelprobe negativ beiderseits. - Kalorische Reaktion beiderseits lebhaft. Drebungsnachnystagmus von gleicher Stärke nach Rechtsund Linksdrehung. - Temp. normal. Befinden ganz gut.

27. Aug. In Äthernarkose Totalaufmeibelung rechts. Warzenfortsatz sklerotisch. Im Antrum und Mittelohr Granulationen und cholesteatomatöse Massen. Tegmen antri et tympani von morschem Knochengewebe gebildet.

Nach der Operation starke Schmerzen im operierten Ohre.

8. Sept. Wăhrend der letzen Tage wiederholte Schwindelanfälle mit Drehempfindungen derselben Art wie vor der Operation. Ist nicht gefallen, kein Erbrechen. Der Schwindelanfall beginnt mit Sausen im operierten Ohre, an welchem sie dann rollig taub zu werden moint. Sausen und Taubheit dauert während des Schwindelanfalles an, verschwinden aber wieder nach dem Aufbören des Schwindels. Kein spontaner Nytagmus.

9. Sept. Entlassen zu poliklinischer behandlung.

16. Sept. Wicuer in dic stationäre Klinik aufgenonmen, wegen andawernder starker Schmerzen im operierten Obr und Schwindels, besonders beim Kehrtmachen nach rechts.

Status praesens: Operations höblo gut granulierend. Funktionsprïfung: Gebör für Flüsterstimme rechts $2.5 \mathrm{~m}$, mit Bárá* ny's Lärmapparat im linken Ohr $0.7 \mathrm{~m}$. Kein spontaner Nystagmus. Fistelp robe negativ. Kal oris che Probe beiderseits positiv mit Drehempfindung. Ophthalmoskopie (19. Sept.) Papillengrenzen verwischt. Linke Papille anscheinend hyperämisch, die Papillen aber kaum geschwollen. Gefäbe nicht gebuchtet. Venen nicht erweitert, keine [lutung, so dab es sich vielleich um eine Pseadoneuritis optica handelt.

2 . Okt. Klagen unverändert. In die neurologische Klinik verlegt.

26. Nov. Zum dritten Male aufgenommen. Nach der Entlassung aus der neurologischen Klinik hat sie fortwăhrend $\$ \mathrm{chmerzen}$ in der rechten Seitedes $\mathrm{N}$ a $\mathrm{ckens}$, aber nur geringen Schwindel. Heute bemerkte sie aber, dafi jhr $8 \mathrm{cb}$ w in d lig beim Blick nach rechts unten wurde; in dieser Blickrichtung auch Doppelsehen. Kein Erbrechen, kein Ohrsausen.

Status praesens: Anr. d. Operationshöhle beinahe völlig epidermisiert. Fn $\mathrm{kt}$ i o n s p ü fung: Ge hö r für Flüsterstimme Aur. a. $0.5 \mathrm{~m}$ (mit Báránys Lärmapparat im linken Obre). Kein spontaner Nystagmus. liesteht gut die gewöhnlichen Gleichgewicht:proben. Kalorische Reaktion positiv. Ophthalmologische Untersuchung weist eine nicht fruber dagewesene Parese des M. obliq. sup. sin., sowie Neuritis optica nach. Neurologis c be Untersuchung ergiebtnichts Abnormes, auber lebhaften tiefen Reflexen.

3. Dez. Temp. 37.7-37. - Gestern und heute Morgen Erbrechen. Klagt uher Schmerzen hinter dem rechten 0 hr. Hier starke Druckempfindlichkeit und etwas pastose Schwellung.

4. Dez. Temp. 37.2-36.6. - Wiederholtes Erbrechen. Ophthalmosk opis ch: Die Verschleierung der Papllen ba tstark augen ommen, ihr Gewebe hyperamisch und ganz lelcht gesehwollen. Die RetinalgefäBe nicht gebuchtet und kaum dilatiert. 
7. Dez. Lumbalpunktur trocken.

9. Dez. Temp. 37,4-37,5. Puls 68-72. Pat. meint, daß ihr Zustand während der letzten Tage sich verschlechtert bat. Der $S \mathrm{ch}$ indel sei stärker geworden, so daß sie einst beinahe die Treppe hinuntergestürzt wäre. Der Schwindel tritt ein, mitunter durch Übelkeit begleitet, wenn sie einige Zeit in rechter Seitenlage verharrt hat, oder wenn sie sich von links nach rechts dreht, oder wenn sie in aufrechter Stellung nach rechts unten blickt, oder endich wenn sie sich aus gebuckter stellung schnell emporrichtet. Er ist von ansgesprochener Drehungsempfindung der Umgebung begleitet (von links nach rechts, nach dem operierten Ohre). Pat. hat ferner die Empfindung nach rechts fallen za sollen, oder, wenn sie in rechter Seitenlage liegt, mehr und mehr nach rechts hinunter zu sinken. Erbrechen einmal heute, einmal gestern. Pat. erzählt ungefragt, daß sie es beinahe nicht verspürt, wenn sie erbrechen soll, nur im letzten Augenblicke. Sie hat keine Übelkeit oder Schwindel vor dem Erbrechen, wohl aber hernach. Das Erbrechen kann im Anschluß zu, aber auch zwischen den Mahlzeiten eintreten. Ohrsausen nur rechts, findet sich sehr häufig ein, wird stärker während der Schwindelanfälle. Kopfwe h andauernd in der Stirngegend, nicht besonders stark, verschlimmert sich nicht während der Schwindelanfälle, wohl aber während (nicht vor) dem Erbrechen. Schmerzen hinter dem rechten Ohre nicht immer zugegen, jetzt am stärksten zur Tageszeit, bat aber früher den Schlaf gestört. Sie werden als hämmernd beschrieben. Die otologische und neurologische Untersuchung ergibt dasselbe Resultat wie am 26. November, doch findet sich jetzt eine ausgesprochene Dr uckempfindlichkeit hinter dem rechten Ohre, am starksten 1. unmittelbar oberhalb des oberen Pols des äußeren Ohres und 2. um das obere Ende der retroaurikularen Narbe herum. Auffallend ist das gesunde, blühende Aussehen der Pat.

12. Dez. Temp. 36.8-36.5. Puls 72-74. Lumbalpunktion trocken. Kraniotomie mit Aufdeckung der Dura in Fossa cranii media et posterior und des Sinus sigmoid. Inzision der Dura und des Gehirns in verschiedenen Ríchtungen, ohne Eiter zu finden. Punktion: (mit Spritze) des Kleinhirns, auch ohne Eiter.

Nach der Operation völliges Wohlbefinden, normale Temp. und Puls, kein Kopfweh oder Schwindel.

19. Dez. Steht auf.

29. Dez. Temp. 37.5-37. Puls $80-88$. - Nenritis optica unverändert. Heute lassen sich keine Doppelbilder mehr nachweisen.

30. Dez. Temp. 37.7-37.1. Puls 104-86. Gestern Kopfweh und sechsmal Erbrechen.

2. Jan. 1909 . Temp. 37.6-37.5. Puls 100-96. Wiederboltes Erbrechen. IBt nichts. Sieht elend aus, bleich, Photophobie. Starkes Kopfweh, mit wesentlicher Lokalisation zur rechten Stirnhälfte.

8. Jan. Temp. 37.5-37. Puls 59-56. 2mal Erbrechen. IBt fast nichts. Am meisten auffallend ist die ausgesprochene Trägheit und Schläfrig keit der Pat. Spontan spricht sie fast nie. Am meisten nimmt sie halbe Bauchlage ein und versteckt das Gesicht in die Kissen. Auf Fragen antwortet sie klar und vernünttig, vielleicht etwas langsamer als normal. Etwas Photophobie. Etwas Kopfweh in der rechten Scbläfengegend. Die neurologis che Untersuchung gibt sonst nichts Abnormes. Durch die Kranitomieöffnung oberhalb des rechten Ohres wölbt sich mehr und mehr ein pulsierender, mit natülicher, behaarter Haut gedeckter, Hirnprolap s hervor. Unterhalb dieser Vorwölbung geht eine kleine Fistel nach oben und vorn in die Gehirnsubstanz hinein.

9. Jan, Temp. 37.1-36.9. Puls 58-64. Operation: Die den Prolaps bedeckende Haut wird losdissekiert, die erwähnte Fistel wird untersucht und aus ihr etwas nekrotisches Hirngewebe entfernt. Danach senkrechte Inzision der Dura und des Prolapses etwa in der Mitte. Hier wird $10 \mathrm{ccm}$ Eiter entleert, derjenigen Stelle entsprechend, wo sie am 11. Dez. Druckempfindlichkeit angab (unter II). Die Abszeßhöhle streckt sich von der Inzision nach hinten und innen, ist von der Oberfache des Gehirns durch 
eine $3 / 4 \mathrm{~cm}$ dicke Schicht Hirnsubstanz geschieden. Der Kleinfinger läbt sich $4^{1 / 2} \mathrm{~cm}$ tief hineinführen und fühlt die Höhle wie mit einem weichen Teppich ausgekleidet. Untersuchung des Hirneiters: Im direkten Präparate als einziger Mikrob in ziemlich reichlicher Menge ein Kettenkokkus. Auf Agar wächst Streptococcus pyogenes.

10. Ja n. Temp. $36.8-37.3$. Puls $64-68$. Befindet sich ganz wohl. Kein Erbrechen oder Schwindel.

13. Jan. Temp. 38.7-37.2. Puls 90-80. Angina. Ophthalmo$\mathrm{k}$ op is $\mathbf{c h}$ : Die Neuritis beiderseits abgenommen, die Grenzen jedoch noch stark verwischt durch weibliches flammiges Exsudat. Die Gefäbe nicht gebuchtet, nur leicht dilatiert. Keine Schwellung der Papillen, keine Hamorrhagien.

18. Jan. Temp. 38.3-37.5. Puls 76-82. Noch Angina. Völliges Woblbefinden. Ist froh und guter Laune. Der Hirnprolaps wölbt sich immer mehr hervor, erschwert sehr die Drainage der Abszeßhöhle.

30. Jan. Temp. 37.2-36.6. Puls $96-86$. Fortdauernde anggesprochene Neuritis optica. Steht auf.

4. Febr. Temp. 37.1-36.8. Puls 92-96. Gestern Abend ein Anfall von starken plötzlichen, "ruckartigen" Schmerzen, von dem Prolaps in die Schläfengegend ausstrahlend. Kein Schwindel oder Erbrechen, aber leichte Übelkeit während des Anfalles.

Während der folgenden Zeit bäufig ähnliche Anfälle. Auch entstand starker Druckschmerz in der Peripherie des Prolapses, besonders nach hinten.

22. Febr. Temp. 37.3-36.6. Puls 88-96. Vielleicht etwas träger als früher. Gestern Schläfenkopfweh wie vor der letzten Operation.

23. Febr. Temp. 37-36.8. Puls 88-96. Äthernarkose. L u m balpunktion entleert $10 \mathrm{ccm}$ klare Flüssigkeit. Sondierung des Fistelganges zur früheren Abszeßhöhle zeígt, daß derselbe durch feste Granulationen geschlossen ist. Kraniotomie hinter der fruher angelegten Ôffnung. In zision der Dura und des Gehirns in verschiedenen Richtungen, obne Eiter. Mikroskopie der Zerebrospinalflüssigkeit: Leichte Vermehrung der Lymphozyten. Keine Mikroben.

24. F ebr. Temp. 36.5-37. Puls 80-92. Ziemlich anhaltendes Erbrechen nach der Operation. Ist schlafrig, aber völlig klar. Der Prolaps sehr abgenommen. Im Verband etwas Zerebrospinalflussigkeit, aber kein Eiter.

27. Febr. Temp. 37.4-36.7. Puls $80-80$. Ne uritis optica unverändert.

1. Mărz. Temp. 37.4-36.8. Puls 80-70. Der Hirnprolaps hat wieder seine frühere Größe erreicht.

3. März. Temp. $36.6-36.6$. Puls $70-68$. Wieder ,ruckartige Schmerzen irn Kopfe.

7. März. Temp. 37.5-37.8. Puls 90-96. Schmerzen hinter dem rechten Ohre; sie werden an einer scharf begrenzten Stelle lokalisiert, hinter und oberhalb des Hautschnittes; daselbst auch starke Druckempfindlichkeit.

16. März. Temp. 37.5-36.6. Puls 90-72. Die Hirnfistel beinahe geschlossen. Der Hirnprolaps bedeutent kleiner. $\mathrm{Oph}$ th al $\mathrm{m}$ o $\mathrm{k}$ op i $\mathrm{ch}: \mathrm{Neu}-$ ritis optica unverändert. Wohlbefinden. Steht auf.

24. März. Temp. 37.9-38.2. Puls 80-88. Wieder ,ruckartige" Schmerzen in der rechten Kopfhälfte. Der Prolaps überhäutet sich gut. Starker Druckschmerz an ihrem hinteren Umfang.

27. Mãrz. Temp. 38.1-38.7. Puls 92-68. Übelkeit und Kopfweh. In der folgenden Zeit unveränderter Zustand mit Ubelkeit, selten Erbrechen, Kopfweb, etwas Photophobie, "ruckartige" Schmerzen in der rechten Kopfhälfte, subfebrile Temperaturen und etwas beschleunigter Puls. Wiederholte neurologische Untersuchung zeigt nichts Abnormes.

20. April. Temp. 37.8-35.6. Puls $80-88$. Neuritis o p tic a uuverändert.

23. A p ril. Temp. 39-38.5. Puls 70-80. Sebr starker Druckschmerz rings der Kraniotomieöffinung, besonders in deren hinteren Abschnitt, Hirnprolaps überhäutet, nur noch walnußgroß. 
25. A pril. Temp. 39.2-39.6, Puls 100-64. In den letzten Tagen sehr trăge, starke Schmerzen in Kopfe. Ätbernarkose. Verschiedene Inzisionen der Dura und des Gehirns an den Stellen, wo sie am stärksten Druckschmerz angegeben batte. Es wurde kein Eiter gefunden. $\mathrm{Lumbalpunktion} \mathrm{trocken.} \mathrm{Drainage.}$

29. A pril. Temp. 37.2-37.2. Puls 70-74. Hat etwas Schmerzen gehabt, befindet sich sonst besser.

30. A p ril. Temp. 39.4-40.2. Puls 104-80. Wiederholtes Erbrechen Zunehmende Schläfrigkeit. Keine meningitische Phănomene. Beim Verbandwechsel wird etwas Fiter in einer der kutanen Inzisionen gefunden. Der Prolaps etwas stărker gespannt mit dunkelfarbigen Sugillationen an der Oberfläche, so daß man eine beginnende Nekrose vermutet. Eine In $z$ i sion des Prolap s e s zeigt mehrere kleine Eiterherde in seiner Substanz. Feuchter Verband.

1. Mai. Temp. 40,6-39.5. Puls 110-120. Sehr stumpfsinnig. Der Prolaps eitrig zerfließend.

2. Mai. Temp. 39.6-39.4. Puls 128-100. Unklar, iBt nichts, involontărer Abgang des Harnes und der Fäces. Paralyse der linksseitigen Extremitäten, keine des Facialis. Kollaps mit Mors um $73 / 4$ Uhr Nachm.

Auszug aus dem Sektionsprotokoll: Grobe Trepanationsöffnung im rechten Schläfenbein, wodurch ein dem rechten Temporallappen gehöriger Hirnprolaps hervorragt. Der Prolaps ist emolliiert und nekrotisch, und die Emollition setzt sich in den Temporallappen hinein fort, beinahe bis zum Frontallappen reichend. Keine Abzeßbildung im Gehirn. Kein Durchbruch nach dem Ventrikel zu.

Subdural und in der Pia in filtriert findet sich rechts eine reichliche Menge dünnen Eiters; muB eher subduraler Abszeb als Meningitis benannt werden. Links weder Meningitis noch Abszeb.

Am Boden der F ossa cranii med, eine chronische Pachymeningitis interna fibrosa, indem hier feine, teilweise vaskularisierte Membranen der zerebralen Seite der Dura anhaften.

Epikrise: Als diese Patientin in unsere Behandlung trat, bot sie objektiv das gewöhnliche Bild einer akut aktivierten, chronischen cholesteatomatösen Mittelohreiterung dar. Unter den subjektiven Beschwerden war auffallen dein seit kurzem bestehendes Schwindelgefuhl, fur welches die genane Untersuchung des Labyrinthes keine Erklärung zu geben vermochte. Die am 17. August 1908 ausgefuhrte TotalanfmeiBelung des rechten Ohres hatte nur für ganz kurze Zeit das Aufhören des Schwindels zur Folge.

Sohon in den ersten Tagen vom September tritt er wieder auf, wird immer in gleicher Weise beschrieben, als eine Empfindung von Drehung der Umgebung von links nach rechts (nach dem operierten Ohre). Der Schwindel hatte insofern einige der Charakteristiken des "Ménièréschen" Schwindels, als er mit Obrensausen begann, und Pat. dann am kranken Ohr taub zu werden meinte. Bei der Unmöglichkeit etwas Abnormes bei der Untersuchung des Labyrinthes zu finden, waren wir geneigt, die Diagnose vermutungsweise auf Morb. Ménière zu stellen. Vielleicht hätten wir auch von Labyrinthreizung, Labyrinthitis serosa oder irgend einer anderen unbestimmten Diagnose sprechen 
können und uns damit zufrieden gegeben. Die richtige Diagnose, Gehirnabszeb, zu stellen gelang uns aber erst während ihres dritten Hospitalanfenthalts, als die ophthalmologisohe Untersuchung unzweideutige Zeichen brachte. Während nämlich früher die Veränderungen im Augenhintergrund als eine mögliche Psendoneuritis anfgefaßt wurde, war jetzt die Neuritis unzweifelhaft, und dazu gesellte sich eine Parese des M. obliq. sup. sin. mit Doppelsehen. Auch wurden jetzt die übrigen Symptome klarer. Die schon früher dagewesenen Schmerzen in der Ohrgegend wurden stark und lokalisierten sich an Stellen hinter dem Ohre, wo starke Druckempfindlichkeit bestand. Es trat Kopfweh auf und Erbrechen von ausgesproehen zerebralem Typus. Der Schwindel bot eine von der Lage des ruhenden Körpers auffallende Abhängigkeit dar, indem er nur bei rechter Seitenlage eintrat, nicht in Rückenlage oder in linker Seitenlage. Es ist dies vielleicht ein Verhältnis, das sich für die Differentialdiagnose zwisehen labyrinthär und zentral hervorgerufenem Schwindel in solchen Fällen verwerteu läßt, in welchen, wie in diesem Falle, das Schwindelgefühl von Nystagmus nieht begleitet ist, und unsere Methoden für die Untersuchung des Vestibularapparates scheinbar normale Reaktionen ergeben. Wir fuhlten uns daher in der Diagnose einer intrakraniellen Komplikation, am wahrseheinlichsten wohl Hirnabszeb, ziemlich sicher und waren nicht wenig enttäuscht, als die Operation am 12. Dezember 1908 uns keinen Eiter finden ließ, weder im Sehläfenlappen noch im Kleinhirn. Die Pat. überstand den Eingriff sehr gut, ihre subjektiven Beschwerden schwanden trotzdem kein Eiter gefunden war, völlig. Daß diese Besserung nur auf die entlastende Wirkung der Operation zu beziehen war, wurde klar, als sie ca. 14 Tagen nachber, wieder Kopfweh und Erbrechen bekam, elend aussah, Photophobie und "slow cerebration" darbot. Auch wölbte sich jetzt ein Hirnprolaps stark hervor, in welchem eine Inzision am 9. Januar 1909 einen Abszeß, durch Streptokokken hervorgerufen, öffinete.

DaB der Sehwindel, das bisher in diagnostiseher Beziehung wohl am schwersten zu dentende Symptom, wirklich von dem Abszeß hervorgerufen war, geht daraus hervor, daß die Pat. nach der Entleerung des Abszesses nicht mehr iber Schwindelgefthl klagte. Wir treten jetzt in die zweite Phase ihrer Krankheit ein, die durch eine, im Anfang langsam, zum Sohluß rapide verlaufende progrediente (Meningo-) Encephalitis gekenn- 
zeichnet ist. Wir haben hier den leider zu gewöhnlicben, entmutigenden Anblick.

Die Encephalitis vergrößert den Hirnprolaps, wodurch die Drainage und das Überwachen der Wunde erschwert wird, und sekundär weitere Möglichkeiten für Verschlimmerung der Encephalitis geschaffen werden. Was man in solehen Fällen tun oder nicht tun soll, ist wahrhaft schwer zu sagen. Wartet man $a b$, geht der Krankheitsprozess erstörend weiter, greift man aber ein, gelingt es nur selten, ein Stillstehen hervorzurufen, und oft stiftet man nur Unheil. So hatten Inzisionen des Hirns am 23. Febr. nur ganz vortibergehend den Prolaps vermindert, er wächst wieder schnell an, und in der folgenden Zeit bis zum Tode der Pat. vermebren sich nur ibre Beschwerden. Als eine akute Verschlimmerung in den letzten Tagen des Aprils zu neuem Eingriff fuhrte, war wobl sehon eine beginnende diffundierende Leptomeningitis da. Zwar wurde bei der Sektion keine makroskopisebe Meningitis links gefunden, aber rechts war reichlich Eiter uber die ganze Hemisphäre verbreitet, sowohl in den subarachnoidalen Räumen, als auBerbalb derselben in Form eines subduralen Abszesses.

Ein interessantes, vortibergehend vorbandenes Symptom war die Parese des linken M. obliq. sup. oculi, die wohl die Diagnose eines basalen pathologischen Prozesses wahrscheinlich machte. Als Ausdruck eines solchen zur Heilung gegangenen Vorganges ist mutmaslich die bei der Sektion gefundene chronische fibröse Pachymeningitis interna der mittleren Schädelgrube aufzufassen.

3. Karen Marie S., (No. 12, 1909) 13 Jahre. Aufgen. 24. Juli 1908, gestorben 12. Januar 1909.

Klinische Diagnose: Otit. med. acut. supp. sin. - Otit. med. chron. supp. dext. - Osteitis acuta et chron. proc. mast. dext. - Abscessus perisinuosus dext. - Thrombophlebitis sinus transv., sin. sigmoid, bulbi venae jugul. int. dext. - Leptomeningitis purulenta diffusa. - Neuritis optica. - Jnvasio bacterialis liq.-cerebrosp.- Prolapsus cerebri. - Abscessus cerebri. - Cystis cerebri. - Encephalitis supp, p. op.

Sektion verboten.

Anamnese. Nach den Mitteilungen der Mutter leidet Pat. seit drei Jabren zeitweise an AusfluB rechts. Während der letzten zwei Monate $a b$ und zu Schwindel und Erbrechen. Por zwei Tagen starke Schmerzen im rechten Ohr. Einmal Erbrechen nuterwegs zur Klinik. Erst heute druckempfindich binter dem rechtem Ohr. Kein Schüttelfrost.

Status praesens: Pat. sieht ganz wobl aus. Temp. 39.2. Puls 100.

A ur. dext: : In der Tiefe des Gehörganges cholesteatomatöse Massen.

Proc. mast. dext: Etwas druckempfindlich. Leichte Infiltration über der Spitze. Aur. sin.: Katarrhalische Veränderungen des Trf. Funktionsprobe: Gehör für Flüsterstimme rechts $0.05 \mathrm{~m}$, nach VerschluB des rechten Gehörganges. Weber nach links. - Leichter spon- 
taner rotatorischer $\mathrm{Nystagmus}$. in extremen Blickablenkungen, besonders deutlich beim Blick nach rechts. Kalorische Vestibularisreaktion beiderseits normal. Fistelp robe beiderseits negativ.

25. Juli: Temp. 39.2-39.8. Unrubiger Schlaf. War gestern etwas benommen. Nachts tiber Nackenschmerzen geklagt. Leichte Nackensteifigkeit. Andeutung von Kernig. Ausgesprochene Druckemptindlichkeit bei Angulus maxillae sowie dem oberen Teil der $V$. jugul. int entlang. Man fuhlt beiderseits deutlicl das Gefälbündel wie einen strang. $0 \mathrm{ph}$ th almos k opie: Neuritis optica beiderseits mitstarker Hyperämie der Retinalrenen und Trubung des Papillengewebes. Um $10^{1 / 2} \mathrm{Ohr}$ vorm. Äthernarkose. Lumbalpunktion entleert $20 \mathrm{ccm}$ unter Druck stehende milchig trube Flüssigkeit Totalaufmeißelung recbts. In den Zellen des Proc. mast. Eiter. Im Antrum und Trommelboble Cholesteatom. Knochengewebe morsch, hyperämisch. Prominentia can. semic. ext. natürlich. An der AuBenwand des Sinus sigmoid. Kiter unter Druck. Der Binus sigmoid. wird in seinew ganzen senkrechten 'Teile aufgedeckt, sowie ein paar cm des angrenzenden Sinus transv. Sinuswand milfarbig, fibrinös besetzt. Pun $k$ tion des Sinus mit Aspiration gibt kein Blut. Resektion der äuB. Sinus wand und Entfernung eines ganz weichen roten Koagulum, wobei Blutung vom Torcular her, aber nicht vom Bulbus trotz gründlicher Ausrăumung des horizontalen Teils des Sinus sigmoid. Abdeckung der Dura der Fossa med. et post. In der Fossa med. ist die Dura stark injiziert. Inzision der Dura in beiden Fossae, Gehirn wölbt sich vor. Inzision des Hirns und Kleinbirns obne Eiter. Jodoformgaze.

Mikroskopie des perisinuösen Eiters: Im Ausstrich eine recht reichliche Menge von Mikroben, teils ein feiner, Gram-negativer Stab, teils und überwiegend Streptokokken, teils endlich Diplokokken verschiedener Größe und Form. Aut Agar wachsen Streptoc. pyog. und Staphyoe pyog. aur. - Mikrosk. des Th rombus: Im Ausstrichpräp. keine Mikroben. Kein Wachstum auf Agar oder in Bouillon. - Sinuswand: Thrombus in beginnender Organisation an der Innenwand einer Vene. - Zerebrospinalfl üssigkeit: Sehr reichliche Menge Leukozyten, überwiegend polynukleäre. Keine Mikroben. Kein Wachstum in Bouilion oder auf Agar nach drei Tagen.

26. Ju1i: Temp. 39.5-39.9-39-37.7. Puls 100-128-104-88. Etwas benommen, befindet sich sonst ganz wohl. Einmal Erbrechen. Nackenund Rückenschmerzen. Kernig beiderseits.

27. Juli: Temp. 38.1-38.3-37.7-37.3. Puls 104-104-104-104.

Wiederholtes Erbrechen. Nackenschmerzen. Geringer Schwindel beim Aufsitzen, bei welcher Gelegenheit auch ziemlich starker spontaner rotator. Nyst nach links beim Blick geradeaus. Etwas Nackensteifigkeit, starker Kernig beiderseits. Verbandwechel. Sinus transversus durch ein Blutkoagulum verschlossen, Sinus sigmoid. leer. Geringer Hirn- und Kleinhirnprolaps. Kalorische Vestibularisreaktion rechts lebhaft. Die Druckemptindlichkeit am oberen Teil der $V$. jugul. int. dext, entlang abgenommen.

28. Juli: Temp. 37.8-37. Puls 80-88. Völlig natürlich. Kein Erbrechen. Lumbalpunktion entleert $15 \mathrm{ccm}$ milchig trübe Zerebrospinalflüssigkeit (vielleicht etwas weniger trübe als die am 25. Juli entleerte). Mikroskopie: Im direkten Präparate eine recht reichliche Menge eines Gram-negativen mit Kapsel versehenen Diplokokkus. AuBerdem in recht reichlicher Menge ein mittelfeiner Gram-negativer Stab. Zahl-reiche Leukozyten, überwiegend mononukleăr. Kein Wachstum auf Agar binnen drei Tagen.

31. Juli: Temp. 37.4-37.9. Puls 80-82. Kaum Nackensteifigkeit mehr, aber starker Kernig. Leichter Nystagmus beim Blicke nach links, rotatorisch nach links.

4. A ug. Temp. 37.5-38. Puls 88-72. Aknte Mittelohreiterung links. Inzision des Trf. Der Hirnprolaps ptaumengrob. Keine Druckemptindlichkeit mebr am Angulus, aber immer noch am vorderen Rande des mittleren Tells des Sternocleido. Kernig gut abgenommen. Lumbalpunktion entleert $3-1$ ccm klare Flüssigkeit. Mikrosk opie: Geringe Menge Lymphozyten, keine 
polynukläre Leukozyten. In ziemlich großer Menge eln mittelfeiner Gramnegativer Stab, der größtenteils Degenerationsphänomene darbietet. Kein Wachstum auf Agar nach zwei Tagen.

12. A ug. Temp. 37.4-37.3. Puls $88-92$. Befindet sich ganz wohl. Kernig fast verschwanden. Lumbalpunktion entleert $15 \mathrm{ccm}$ völlig klare Flüssigkeit. Mikrosk o p is $\mathrm{ch}$ : Ziemlich reichliche Menge Lymphozyten, keine polynukleäre Leukozyten, keine Mikroben. Wachstum auf Agar von Staphyloc. pyog. albus.

1.7. A ug. Temp. 37.5-37.2. Ophthalmoskopie: Die Neuritis bedeutend abgenommen, bes. rechts, wo die Verhältnisse fast natürlich sind.

27. Aug. Temp. 37-36.6. Noch Andeutung ron Kernig. Da der Hirnprolaps andauernd zunimmt, entscheidet man sich, obgleich sonst keine $\mathrm{Ab}$ szeßsymptome da sind, zu dessen Inzision, nm nicht einen Abszeß zu ubersehen. A thernarkose. Lumbalpunktur entleert $10 \mathrm{ccm}$ klare Flüssigkeit Mikroskopie: Recht sparsame Menge hauptsächlich polynukleäre Zellen. Außerdem in direktem Prăparate verschiedene Mikroben: ein Stab und ein kleiner Gram-positiver Diplokokkus. Kein Wachttumin Bonillon der auf Agar nach 18 Stunden. - Inzision des Prolapses an drei Stellen; es wird nur Blut entleert.

25. Aug. Temp. 37.8-37.3. Pals 94-84. Woblbefinden seit der Operation.

6. September: Temp. 37.7-36.9. Puls 112-100. Kein Kernig mehr. Der Hírnprolaps nimmt langsam, aber stetig zn.

17. Sept. Temp. 37.2-36.8. Pals $100-104$. Lu mbalpunktion entleert $15 \mathrm{ccm}$ klare Fltssigkeit. Mikroskopie: Ganz vereinzelte Lymphozyten, keine Mikroben. Kein Wacbstum auf Agar nach 48 Stunden. Ophthalmosk opie: Die Neuritis stärker geworden; bedeutende Hyperämie und leichte Schwellung des Papillengewebes. Frische Hämorrhagien an den Papllen und in deren Umfang. Harn o. B.

19. Sept. Temp. 37.3-37.2. Puls 106-100. Während Pat. sich sonst lange sehr wohl befunden hat, trat gestern einmal Erbrechen auf. Keine weiteren zerebralen oder zerebellaren Symptome. Kein Kernig. Kein spontaner Nystagmus. Gehör für Flüsterstimme rechts 0.05 , mit verschlossenem rechtem Gehärgang 0 . Kalorische Vestibularisprüfung rechts ne. gativ (zwischenliegende, dem Prolapse gehörige, Hirnmasse?).

20. Sept. Temp. 37.5-38.3. Pals $11 \%-120$. Nach der Kaltwasserspülung des rechten Ohres gestarn Schmerzen in der rechten Schläfengend und Schwindel. Wiederholtes Erbrechen. Kein spontaner Nystagmus, keine Nackensteifigkeit, aber zweifellos leichter Kernig, am stärksten rechts.

21. Sept. Temp. 37.7-37.4. Puls 106-108. Völliges Woblbefinden.

25. Sept. Temp. 37.2-37.1. Puls 100-108. Befand sich gestern wieder schlecht, klagte über Schmerzen in der rechten Schläfe. Etwas Drackemptindlichkeit der rechten Kopfhälfte. Hirnprolaps stark gespannt. Schwacher Kernig. Athernarkose. Punktion des Hirnprolapses in der Richtung von Cerebrum und Cerebellum. Kein Eiter, aber an einer Stelle etwas blutig seröse Flüssigkeit entleert.

26. Se p t. Temp. 39.2-39.2. Puls 120-120. Nacb der Operation ziemlich viel Schmerzen, einmal Erbrechen. Heute morgen diffuser Kopfschmerz.

27. Se p t. T e m p. 38.9-39.2. Puls 128-124. Gestern recht starke Nacken- und Schläfenschmerzen. Photophobie. trocken.

29. Sept. Temp. 37.6-37.8. Puls 100-100. Lum balpunktion

5. Oktober. Temp. 38.5-37.8. Puls 100-98. Zeitweise etwas benommen. Sieht $a b$ und zu schlecht aus. Heute sieht man beim Verbandwechsel, daß Eiter ziemlich reichlich an der medialen hinteren Seite des Prolapses hervorströmt. Es gelingt nicht, die Ausflußöffnung zu finden.

6. Okt. Temp. 38.5-37.6. Puls 94-88. Athernarkose. Lumbalpunktion entleert $15 \mathrm{ccm}$ klare Flüssigkeit. MikroskopiB: Im direkten Präparate Lymphozyten, vielleicht etwas reichlicher als normal. In sparsamer Menge ein mittefeiner Gram-negativer Stab. Kein Wachstum 
auf Agar nach 48 Stunden. - Danach In zision des Prolapses mit Entleerung erst seröser, dann eitriger Flàssigkeit.

8. $0 \mathrm{kt}$. 'lemp, 37.8-37.4. Puls 112-105. Woblbefindeu. Hirnprolaps kleiner und weniger gespannt.

17. Okt. Temp. 37.4-36.5. Puls $88-84$. Steht auf. Kein Kernig.

2. November. Temp. $37.6-36.7$. Puls $96-84$. Oph thalmoskopie: Bedeutende Neuritis optica. Papillen stark hyperämisch, etwas geschwollen (3-4 Dioptrien) mit vereinzelten feinen Hămorrhagien.

30. Dezember. Neuritis optica auch jetzt bedentend mit starker Hyperämie. Schwellung doch nur 2 Dioptrien

31. Dez. Temp. 37.4-36.6. Pat befindet sich seit langem ausgezeichnet. Der Prolaps ist noch immer gro $\beta$ und seine am meisten prominierende Partie scheint sich nicht überhänten zu wollen. Fin Teil des Prolapses findet sich auch im Antrum und Mittelohr, so dab man die Operationskavität nicht überseben kann. Es wird Punktion des Prolapses mit Aspiration vorgenommen. In einer Tiefe von ca. $1.5 \mathrm{~cm}$ werden einige Kubikzentimeter trübe Flüssigkeit aspiriert. Mikros ko pie: Enthält nur vereinzelte, undeutlich konturierte Zellen. Keine Mikroben. Kein Wachstum auf Agar und in Bovillon nach 18 Stunden.

1. Januar 1909. Temp. 37.4-39.1. Pat. befand sich gestern den ganzen Tag wohl, hat aber unrubig geschlafen. Klagt heute Morgen aber Ohrschmerzen rechts. Kein Kopfweh. Kein Erbrechen. Hirnprolaps zugenommen, seine Haut rot und heib.

2. Jan. Temp. $39.7-39.5$. Puls 140-128. Röte und Schwellang des Prolapses bedentend zugenommen. Befinden schlechter. Äthernarkose. Lumbalpunktion entleert $2 \mathrm{~cm}$ völig klare Flüssigkeit. Mikrosk opie: Lymphozyten etwas vermehrt. Keine polynukleäre Zellen. In geringer Menge ein Gram-positiver Diplokokkus obne Kapsel, nirgends in Ketten geordnet. Auberdem in reichlicher Menge ein mittelfeiner, ziemlich langer Gram-negativer Stab. - Darnach Inzision des Prolapses. Man findet Eiter, sowohl in eincr subkutanen als in eincr tiefer liegenden Höhle. Aulerdem zeigt sich die Schnittfläche uberall mit kleinen Fiterherden durchsetzt. Mikrosk opie des Eiters: Gram-positive Streptokokken.

3. J a n. Temp. 30-37.t. Puls 120-102. Befindet sich besser. Kein Kernig oder Nackensteifigkeit.

4. J a n. Temp. 39.8-40.1. Puls 120-120. Hautig benommen. Klagt über Stirnkopfschmerzen. Kein Erbrechen. Keine Nackensteifigkeit, aber ausgesprochener Kernig. Der Prolaps făngt an zu gangräneszieren.

5. J a n. T e mp. 40-39.9. Puls 120-112. Sehr unrubig, schreiend. Seufzen. Keine Nackensteifigkeit.

9. Ja n. Temp. 39.4-40.5. Puls 106-112. Klagt aber Schmerzen in der Wirbelsäule und im Nacken. Lumbalpunktion entleert $5 \mathrm{ccm}$ trübe Flüssigkeit. M i k r os k o p i e: Im direkten Präparate zahlreiche, hauptsăchlich polynukleäre Leukozyten und in reichlicher Menge ein Gram-po sitiver Diplokokkus, teilweise mit Kapsel versehen. Auf Agar wachsen Pneumokokken in Reinkultur.

11. J a n. Temp. 40.3-40.3. Puls 124-120. BewuBtlos. Unreinlich.

12. J a n. 40.2-42. Puls $123-64$. Starb $7 \frac{1}{2}$ morgens.

Sektion verboten. Der Prolaps wurde postmortell exstirpiert, war halbflüssig, enthielt aber keinen gröBeren Hohlraum. Von den Kraniotomieöfinungen aus konnte man keinen $A$ bszef im Cerebrum oder Cerebellum finden.

Epikrise. Bei diesem Falle wäre zu erörtern erstens unsere Diagnose bei der Aufnahme, zweitens der Verlauf und endlich der unglückliche Ausgang der Krankheit.

Bei der am Morgen nach der Aufnahme vorgenommenen Operation wurde ein perisinuöser Abszeß und Epiphlebitis des Sinus sigmoideus gefunden. Vom senkrechten Teile des Sinus sigmoid. 
wurde ein ganz weicher, roter Thrombus entfernt, aber der primäre Herd hat wahrscheinlich seinen Sitz im Bulbus gehabt, da der entfernte Thrombus sich als steril erwies, und man keine Blutung vom Bulbus hervorzurufen vermochte. Auch war ziemlich starke Druckempfindlichkeit in der Fossa retromaxill. und dem oberen Teil der V.jugul. int. entlang vorhanden, so dab wohl auch eine weniggtens partielle Thrombose der V. jugul. int. vorlag. Diese Komplikation ging, nachdem Eiterung ans dem horizontalen Teil des Sinus sigmoideus einige Zeit bestanden hatte, zur Heilung, ein Beweis unter mehreren dessen, daß eine Bulbusthrombose ohne Bulbusoperation ausheilen kann.

Wir haben ferner bei der Aufnahme die Diagnose Leptomeningitis purulenta diffusa gestellt und stützten uns dabei auf die Temperaturkurve, das Vorhandensein von Nackensteifigkeit und Kernig, welche Symptome in den folgenden Tagen noch stärker hervortraten und erst langsam schwanden, - Kernig war nach 6 Wochen noch vorhanden, - sowie endlich auf das Ergebnis der Lumbalpunktion. Die Zerebrospinalflissigkeit war nämlich milebig trube und enthielt eine sehr reichliche Menge, hauptsächlieh polynukleärer Leukozyten. Da die Flüssigkeit bei dieser ersten Punktion steril war, wird man vielleicht von verschiedener Seite Einspruch gegen die Bezeichnung des Falles als Leptomeningitis purulenta diffusa erheben. Dem gegentaber wünschen wir erstens daranf aufmerksam zu machen, daB die Sterilität der entleerten Flüssigkeitsmenge natürlich keinen zwingenden Beweis dafür abgibt, daß Mikroben sich überhaupt nicht in der Zerebrospinalflussigkeit befinden. Und betrachten wir die Frage vom klinischen Standpunkt aus, wird es uns doch natürlicher scheinen, Fälle, die wie dieser dureh typisebe meningitische Symptome und eine durch (hier polynukleäre) Leukozyten verursachte Trübung der Zerebrospinalflüssigkeit charakterisiert sind, Leptomeningitis purulenta diffusa zu benennen, als sie außerhalb dies nosologischen Begriffes anbringen zu wollen. Die Gegner einer solchen Betrachtungsweise machen gewöhnlich auf den vermeintlich prinzipiellen Unterschied zwischen den zwei Klassen von Erkrankungen aufmerksam: In der einen seien Mikroben in der Zerebrospinalflüssigkeit vorhanden und wirken daher direkt anf die Meningen ein, in der anderen sei die Auswanderung von Leukozyten nur indirekt durch Toxinwirkung hervorgerufen. Nun, unsres Erachtens ist es doch nur ein und derselbe ProzeB, der in beiden Klassen in der Leukozytenauswanderung resul- 
tiert; das wirksame Prinzip ist in beiden Fällen die chemotaktische Eigenschaft der in der Zerebrospinalflussigkeit zirknlierenden Toxine, und der pathologiseh-anatomische Befund in den Hirnhäuten wird wohl auch in den Hauptzligen derselbe sein. Damit wollen wir durchaus nicht bestreiten, dab in prognostisoher Beziehung ein Untersehied zwisehen den zwei Klassen zu existieren scheint.

Während, wie erwähnt, die bei der ersten Punktion gewonnene Zerebrospinalfüssigkeit keine Mikroben entbielt, fand man bei der 3 Tage später vorgenommenen zweiten Punktion Diplokokken und Bakterien im direkten Präparate der trüben Flüssigkeit. Daß dies nicht eine Versehlimmerung des Zustandes bedeutete, scheint sowohl aus dem klinischen Verlauf-als daraus hervorzugehen, daß die zytologische Formel überwiegend mononukleär geworden war.

Wir wollen daher auch nicht behaupten, daß diese Mikroben wahrseheinlich sehon bei der ersten Punktion in derZerebrospinalflikssigkeit vorhanden waren und nur nieht nachgewiesen wurden, sondern geben gern die Möglichkeit zu, dab es sieh bei ihrem späteren Vorhandensein um eine Invasion nicht besonders pathogener Mikroben bandele.

Die Möglichkeit einer solchen Invasion war ja durch die bei der ersten Operation ausgeführte Durainzision gegeben. Für diese Deutung spricht wohl auch der wiederholte spätere Fund von Mikroben in sonst anseheinend normaler Punktionsflüssigkeit (am 4. August und 27. Angust).

Fragt man nach der Ätiologie der Leptomeningitis im vorliegenden Falle, so kann man sie wohl jetzt, da der ganze Krankheitsverlauf uns vorliegt, mit ziemlicher Sicherheit auf die Sinus- und Bulbusthrombose beziehen. Bei der ersten Operation mußte man auch andere Möglichkeiten erwägen, z. B. die eines Hirnabszesses - starke Neuritis optica! - und es wurden daher Inzisionen im Groß- und Kleinhirn gelegt. Es wurde kein Eiter gefunden, und es war vielleicht anch kein AbszeB da. Etwa 10 Wochen später wurde zwar ein AbszeB im Hirnprolaps inzidiert, aber möglich ist es immerhin, daß dieser durch unsere Eingriffe bei der ersten Operation oder später (27. Aug., 25. Sept.) verursacht worden ist. Man könnte daher vielleicht die Regel aufstellen, daß man bei der Operation von Leptomeningitiden nur dann die Gehirnsubstanz inzidieren soll, wenn ein Gehirnabszeß sich mit verhältnismäßig großer Sicherheit diagnostizieren läßt, 
oder wenn man bei der Untersuchung und im Laufe der Operation kein Leiden nachweisen kann, das als primärer Infektionsherd gelten könnte (z. B. Sinusthrombose, Labyrinthitis, Pachymeningitis externa oder interna).

Der Verlau f der Krankheit steht ganz im Zeichen des Hirnprolapses. Es hat für den behandelnden Arzt etwas Unheimliches an sich, diesen Ausläufer des Hirns langsam aber stetig wachsen zu sehen, ohne dab es ihm möglich ist zu wissen, weiche pathologischen Prozesse in dessen Innern sich abspielen. Dieses Wachstum und der immer noeh bedeutende Grad der Neuritis optica machten uns befurchten, einen Abszeß zu thersehen, weshalb wir am 27. August den Prolaps inzidierten, ohne Eiter zu finden. Der Eingriff schien zuerst keine schlimmen Folgen zu haben, im Gegenteil; so sohwand der noch am 27. August vorhandene Kernig völlig. Aber am 17. September war die Neuritis optica stärker geworden, am nächsten Tage trat Erbrechen auf, noch später wurde der Hirnprolaps stark gespannt und nach einigen am 25. September vorgenommenen Punktionen des Prolapses, wobei an einer Stelle etwas blutig seröse Flüssigkeit entleert wurde, stieg die Temperatur stark an, und der Zustand wurde ziemlich sehlecht und besserte sich erst, nachdem ein AbszeB sich von selbst eröffnet hatte und darauf am 7. Oktober drainiert worden war. Es ist naturlioh unmöglich, bloß einigermaßen mit Sicherheit zu behaupten, daß die Verschlimmerung durch unsere Eingriffe vom 27. August und 25. September verursacht worden war, aber der Verlauf gibt zweifellos viel zu denken, besonders imLichte der unglioklichen Folgen eines späteren Eingriffes gesehen.

Der weitere Verlauf war während beinahe drei Monaten durch das denkbar beste Befinden gekennzeichnet, und die starke Neuritis optica ging auch etwas zurück, insofern als die Schwellung der Papillen von 3-4 anf zwei Dioptrien herabging. Aber immer noch war die Neuritis ausgesprochen, der Prolaps groß und wollte sich nicht völlig tiberhäuten. Bei einer Punktion des Prolapses am 31. Dezember wurden einige Kubikzent. trübe Flussigkeit aspiriert, deren mikroskopische Untersuchung nachwies, daß sie wohl aus einer Zyste stammte (Residunm des am 7. Oktober geöffneten Abszesses?). Unglücklicherweise trat, trotz den wie gewöhnlich beobachteten strengen anti- und aseptischen Kautelen, eine Infektion des Prolapses mit Streptokokken ein; und es entwickelte sich jetzt erst eine eitrige und gangrä- 
nöse Enzephalitis und kurze Zeit nachher eine eitrige Pneumokokken-Meningitis. Spaltung des Prolapses blieb ohne dauernden Erfolg.

Sollten wir aus dem Verlanf dieses Falles eine Lehre fur die Zukunft zu ziehen suchen, so scheint der Fall uns zu zeigen, daß man Hirnprolapsen gegentiber sich am besten konservativ verhält, wenn nicht gefahrdrohende Symptome uns das Messer in die Hand drücken, und daß man sich von der Besserung einer etwa bestehenden Neuritis optica in dem konservativen Verfahren bestärken lassen soll. Entscheidet man sich aber für ein aktives Einschreiten, dann sei das Messer und nicht die Punkturspritze unser Helfer. Es muß sicher als anßerordentlich schwierig betrachtet werden, die Oberfläche eines im Epidermisieren begriffenen Hirnprolaps zu desinfizieren, und wenn dem so ist, dann ist doch wohl die Gefahr, durch eingebrachte Infektionserreger Unheil zu stiften, unvergleichbar kleiner bei offenen Inzisionen als bei Punktionen.

4. Jens Peter J., (No. 13, 1909) 29 Jahre, aufgenommen 6. Jan. 1909, gestorben 15. Jan. 1909.

Klinische Diagnose: Otit. med. chron. supp. dext. - Osteit. acuta capsul. labyrinth. dext. - Abscessus epiduralis. - Otitis interna acuta supp. diffusa. dext. p. op. - Leptomeningitis purul. diffusa p. op.

Sektionsdiagnose: Neningitis purulenta diffusa. - Hyperaemia et Oedema pulmonum. - Putrefactio organorum.

A namnese: Seit den Kindesjahren zeitweise Ohrflußs und Ohrschmerzen rechts. Während der letzten drei Wochen andauernde reichliche Eiterung sowie ziemlich starke Schmerzen. Scbwindel nicht ausgesprochen, kein Erbrechen, aber etwas Übelkeit. Empfindlich starken Geräuschen gegenüher.

Status praesens: A ur. dext. Im Gehörgang Schleimeiter obne Geruch. Trommelfell hellrot, etwas geschwollen, runde Perforation nach vorn, durch welche der Eiter pulsierend hervordringt. Inz is ion des Trf. - Proc. mast d ext.: Ziemlich starker Druckschmerz, besonders über Fovea, wo auch etwas Infiltration. - A u r. si n. Katarrbaliscbe Veränderungen des Trf. - Funktio n sprüfnng: Gehōr für Flüsterstimme recbts $0.25 \mathrm{~m}$ (mit Báránys Lărmapparat im linken Ohr). - Kein spontaner Nystagmus. Kalorische Prüfung beiderseits normal.

Da die Ohrschmerzen recht heftig andauerten, und Granulationen aus einer Antrumfistel hervorsprießten, wurde (bei normaler Temperatur) am

12. Januar die Totalaufmeißelung rechts ausgefübrt. Das Knochengewebe des Warzenfortsatzes ziemlich fest, obne gröbere osteitische Veränderungen. Antrum klein, tief und hoch liegend, mit Granulationen und Detritus gefüllt. Dura wird in der Fossa cranii med. etwas aufgedeckt, scheint normal, wie auch die Sinuswand. Der Hammer wird entfernt, ist anscheinend normal, der AmboB nicht gefunden. Prominentia canal. semicirc. ext. ist nicht wie gewöhnlich, weiß und glatt, sondern rötlich und uneben. Promontorium natürlich.

13. Jan. Temp. 38-38.6. Kein Erbrechen während der ersten zwölf Stunden nach der Operation, dann aber ein paarmal Erbrechen. Keine Klagen über Schwindel. Heute Morgen Stirnk opf schmerzen. Wenn Pat. im Bett aufrecbt sitzt, hat er die Empfindung nach links, seltener nach rechts fallea zu sollen. Keine Rotationsempfindung. Keine Nackenstarre, doch gibt Pat. an, in den Nackenwirbeln 
S c h merz zu fäblen, wenn er den Kopf nach vorne beugt. Kein Kernig. Links ist das Zeben phänomen dorsalen Typus. Oppenheim.dorsal beiderseits. - Ziemlich starker spontaner Nystagmus, am deutlichsten beim Blick nach links, wesentlich rotatorisch nach lin $k s$; recht hăufig ist derselbe Nystagmus anch zu beobachten beim Blicke geradeaus, ja selbst beim Blick nach rechts. Nach Entfernung der Tamponade Prâfung des Gebörs: Flästerstimme wird rechts nicht mehr gehört, Konversationstimme rechts im Abstand $0.5 \mathrm{~m}$, aber mit Báránys Lärmapparat im linken $\mathrm{Ohr}$ werden selbst laute Rufe nicht gehört. - Ophthalmoskopie normal.

Dem Pat. wurde vergebens Labyrinthoperation vorgeschlagen.

14. Jan. Temp. 39.6-39.2, Puls 90-88. Kein Erbrechen. Stirnkopfschmerzen abgenommen, Nackenschmerzen wie gestern. - Heute morgen unzweifelhaite $\mathrm{N}$ ackensteifigkeit und beginnender Kernig beiderseits, Prüfung des Gehörs: wie gestern totale Taubheit rechts. Kalorische Reaktion (Wasser $48^{\circ} \mathrm{C}$ ) negativ. Nach langem Verweigern willigt Patin eine zweite Operation ein. Athernarkose. Lumbalpunktur entleert $10 \mathrm{ccm}$ trübe Zerebrospinalfüssigkeit. Nach Öffnung der gut agglut tinierten retroaurikulären Inzision findet man die Operationskavität gu . aussehend. Nur gibt es, am binteren Abschnitte des Can. semic. ext. herum eine akut osteitisch veränderte Knochenstelle mit Granulationen. Mit Sonde läßt sich hier keine Fistel nachweisen. Es werden jetzt der Sinus und die Dura fossae post. zwischen Sinus und Porus acust. int. aufgedeckt. Beim Vordrăngen in letztgenannte Region entleert man eine kleine epidurale Eiteransammlung. Resectio auris internae, wobei von der Spitze des Sporns ein Knochenstack losgesprengt wird, das auf seiner tiefen Seite eine Halbrinne vermutlich der AuBenwand des Can facialis entsprechend) enthält. Der Steigbügel ist wegen Granulationen nicht zu sehen, sitzt aber scheinbar fest im ovalen Fenster, woselbst die Sonde recht festen Widerstand findet, der beim fortgesetzten brucke plötzlich nachgibt. Jetzt werden die Bogengänge weggemeißelt, so daßs man ins geöffnete Vestibulum von oben hineindringt und die Sonde wieder durch das ovale Fenster ins Mittelohr hinausfubren kann. Im Vorhof kaum Eiter. Kraniotomie, Inzision der anscheinend normalen Dura in Fossa cranii med. et post. mit Entleerung von ziemlich reichlicher Zerebrospinalflüssigkeit. M i k r oskopie der Zerebrospinalflüs sigkeit: Sehr zahlreiche monound polynukleäre Leukozyten nebst Gram-positiven Diplokokken obne Kapsel, teilweise in kurzen viergliedrigen Ketten geordnet.

15. Jan. Temp. 39.4-39.5. Pals $80-76$. Sofort nach der Operation wurde gestern eine Parese des rechten Facialis beobacbtet. Nystagmus wie vor der Operation, nicht.stärker. Kein Erbrechen oder Schwindel. Keine Rotationsempfindung. Starke Kopfschmerzen. Während der Nacht benommen. Starb heute Morgen um $91 / 2$ Uhr.

Epikrise: Dieser traurige Fall von postoperativer Leptomeningitis ist in wissensehaftlicher Beziehung darum von Interesse, weil die klinische Beobachtung uns den durch die Infektion eingeschlagenen Weg sehr klar vor die Angen führt. Der Pat. hatte eine chronisohe Mittelohreiterung, während der letzten Zeit mit starken Schmerzen und Andeutung von Schwindel kompliziert. Die Untersuchnng vor der Operation zeigte, daß sow ohl Cochlea als Vestibularapparat funktionierte. Die Operation selbst gab keinen rechten Aufschluß bez. der starken Schmerzen, indem der pathologische Prozeh die Grenzen des Antrums nicht überschritten zu haben schien; anch wurde kein Epiduralabszeb gefunden, obgleich Dura und Sinuswand etwas 
entblöBt wurden. Dagegen war das pathologiseh veränderte Aussehen der Prominentia can. semic. ext. auffallend. Eine am Abend des Operationstages einsetzende Verschlimmerung des Zustandes des Pat. ließ schon am nächsten Morgen eine sehr ernste Wendung seiner Krankbeit befürchten. Unter ansteigender Temperatur und von Erbrechen eingeleitet, waren starkes Kopfweh und spontaner Nystagmus, hinzugetreten. Die Besohaffenheit des Nystagmus, rotatoriseh nach der gesunden Seite schlagend, deutete eine Destruktion des Vestibularapparates des operierten Ohres an, welche Diagnose durch die Unmöglichkeit, den spontanen Nystagmus mittelst Heißwasserspülung der Operationskavität zn beeinflussen, bestätigt wurde. Die Hörprüfung ergab an der operierten Seite totale Taubheit, so dab auch deren akustische Funktion zerstört worden war. Damit war die Diagnose einer postoperativ cingetretenen diffusen Otitis interna gegeben. Fraglich war nur noch, ob sich bereits eine Leptomeningitis dazu gesellt hatte. Es waren zwar keine der klassisehen Zeiehen da, aber die - bis jetzt immerhin moderate - Temperatursteigerung $(38-38.6)$ und die Sebmerzen in Nackengegend legten die Befürohtung sehr nahe. Wir rieten daher mit gewissenhafter Darlegung des Funstes der Situation, dem röllig klaren Pat. eine neve Operation an. Hätten wir seine Linwilligung erhalten, würden wir vorerst das Labyrinth drainiert haben, falls aber die Zerebrospinalflïssigkeit noch normal war, uns weiterer Eingriffen enthalten. Als aber der Pat. endlich einwilligte, war die diffuse Leptomeningitis 48 Stunden nach der Totalaufmeißelung klinisch unzweifelhaft nachweisbar. Bei der zweiten Operation fanden wir eine umschriebene akut osteitische Veränderung des Knochengewebes des Pars petrosa, an die schon erwähnte Osteitis des Prominentia can. semic. ext. grenzend, sowie in deren Nähe einen tiefliegenden epiduralen kleinen Eiterherd, der die initialen Ohrselmerzen des Pat. verursacht haben mag. Eine etwaige, bis in das Innere des Labyrinthes futhrende Fistel war nirgends nachzuweisen; auch sehien der Steigbtigel fest an normaler Stelle zu sitzen. Dies Verhältnis macht an sich, wie bekannt, keine Sehwierigkeiten für die Erklärung des Entstehens der Otitis interna, da jetzt ziemlich zahlreiche Fälle bekannt sind, in welohen die Infektion des Labyrinths ohne gröbere Läsionen der Labyrinthkapsel mikroskopisch nachgewiesen worden ist. Die Labyrinthoperation und Drainage des Sub- 
duralraumes waren jetzt leider nicht imstande, den Pat. zu retten. Vielleicht wären unsere Bemühungen von besserem Erfolg gekrönt gewesen, falls wir - wie wir es wollten - die Labyrintheröffnung 24 Stunden früher hätten vornebmen können.

In Betracht des Befundes bei der zweiten Operation sowie des Umstandes, daß keine Retention durch die Tamponade hervorgerufen zu sein schien, meinen wir zwar, daß dieser Todesfall durch eine postoperative Otitis interna und Labyrinthitis verursacht ist, aber gleichzeitig daß er kaum einem Kunstfehler unsererseits zuzuschreiben ist. Die natïrliche Infektionsquelle scheint uns mit der nachgewiesenen akuten Osteitis der Labyrinthkapsel gegeben zu sein. Endlich mag vielleicht auch der epidurale Abszeß für das Entstehen der Leptomeningitis von Bedeutung gewesen sein.

5. Olga Marie L., (No. 107, 1909) 14 Jahre, aufgenommen 1. April 1909, gestorben 12. April 1909.

Klinische Diagnose: Otit. med. chron. supp. dext. et sin. - Osteit. acut. in proc. mast. sin. - Abscessus perisinuosus sin. - Epiphlebitis sin. sigmoid sin. - Thrombophlebitis sin. sigmoid., bulbi v. jugul. int. sia. - Abscessus epiduralis sin. - Pachymeningitis ext _ Otitis interna serosa sin? - Otit. interna. diffusa acuta sin. p. op. - Leptomeningitis diffusa purul. p. op. - Prolapsus cerebri.

Sekti onsdiagn ose: Meningitis purulenta diffusa. - Otit med. purul.

A nam nese: In den letzten 6 Jahren Ohrflul beider seits (nach Scarlatina). Vor vier Wochen wurde ein Polyp aus dem linken Obr extrahiert. Während der letzten 4 Tage wiederholtes Erbrechen. Etwas schwindlig. Keine Obrschmerzen, kein Öhrsausen.

Status praesens: Gesundes Aussehen. Temp. 37.4. Púls 104. A ur. dext.: lo Gehörgang weiße, fettige Massen, welche genaueren Einblick verhindern. A ur. sin : Stinkender Eiter. In der Tiefe des Gehörganges Polypen, von hinten oben ausgebend. - Regiones mast: 0 . $B$. F unktionsprüfung. Gehor für Flüsterstimme r. $2.0 \mathrm{~m}, 1.1 .5 \mathrm{~m}$, mit Báránys Lärmapparat im anderen $O b r$ beiderseits $0,5 \mathrm{~m}$. Weber nach beiden Ohren. - Kein spontaner Nystagmus. Etwas schwindlig, geneigt nach links $\mathrm{zu}$ fallen. Kaloris cher $\mathrm{Ny}$ stag m u beiderseits sehr schnell und stark eintretend, von ausgeprochenem Schwindel und etwas Erbrechen begleitet.

2. A pril. Temp. 37.9-37.7. Beim Aufstehen heute morgen schwindlig. Bei der Visite bettlägerig, mit Ubelkeit und Schwindel: die Umgebung dreht sich, wie immer, von links nach rechts. Kein spontaner Nystagmus. Fistel p ro b e ruft keinen Nystagmus vor. Pat. sagt zwar, daß ihr bei der Kompression, nicht bei der Aspiration, schwindlig wird, und daB die Umgebung dann von rechts nach links dreht, aber sie hat dieselbe Drehungsempfindung bei der Untersuchung sowohl des rechten als des linken Ohres,

3. April. Temp. 38-37.3. Äthernarkose. Totalaufm e i ß e lu ng links. Beim ersten Meißelschlag wird eine mit pulsierendem Eiter gefüllte Höhle geöffnet, deren Hinterwand von dem granulationsbesetzten senkrechten Teil des Sinus sigmoid. gebildet wird. Die ganze Spitze des Proc. mastoid. muß entfernt werden. Antrum mit Granulationen gefüllt. An seiner Medialwand findet sich eine Stelle mit sehr morschem Knochengewebe; sie wird so tief ausgeloffelt, als man des hinteren Bogenganges wegen vordringen darf. Prominentia can. semic. ext. natürlich. Dura nicht aufgedeckt. 
4. A p ril. Temp. 39.3-38-39. Puls 123-113-114. Kein Erbrechen gestern nach der Narkose. Gestern Abend kein spontaner Nystagmus. Nachts um 1 Uhr Erbrechen, spăter viermal mit Drehempfindung. Bei der Visite gibt sie an, dab das Bett sich mit ihr dreht, teils um eine senkrechte Achse, teils um eine wagerechte Längenachse, so daß sie die Empfindung hat, aus dem Bette herausstürzen zu sollen. Aussehen nicht scblecht Keine Fazialisparese. Starker spontaner horizontaler und rotatorischer $\mathrm{Nystagmu}$ s nach rechts (dem nicht operierten Ohre), am stärksten beim Blick nach rechts, aber vorbanden selbst beim Blick nach links. Verbandwechsel. Der Verband mit blutiger, nicht seröser Flüssigkeit durchtränkt. Nach Entfernung der Tamponade W eber von Stirn und Scheitel aus nach beiden Obren, vom Kinn aus nach dem rechten, nicht operierten Ohre. Gehör für Flüsterstimme links 0. für Konversationssprache links 1, mit Báránys Lärmapparat im reehten Ohre 0. - Ausspalung des linken Ohres mit $47^{\circ} \mathrm{C}$ warmen Wasser beeintulit nicht den bestehenden Nystagmus, - Keine Nackensteifigkeit. Ganz leichter Kernig. Ophthalmoskopie o. B. - Athernarkose: Lumbal punktion entleert $10 \mathrm{ccm}$ ziemlich stark getrübte Flüssigkeit. Mikroskopie: Reichliche Menge mono- und - hauptsächlich - polynukleäre Leukozyten. Keine Mikroben. Kein Wachstum anf Agar oder in Boullon. - Inspektion deroperations höh le: Prominentia can semic. ext. sieht gut aus, am Promontorium nichts Abnormes. - Krani ot om ie: Oberhalb des Tegmen antri ein nicht ganz kleiner epiduraler Abszeb; Dura hier mit dicken mißfarbigen Granulationen besetzt. Mik ros k o pi e des Eiters: Reinkultur von Streptokokken - Die bei der ersten Operation gefundene morsche Knochenstelle an der Medialwand des Antrum wird völlig ausgelöffelt, scheint nicht in Verbindung mit Bogengăngen oder Vestibulum zu stehen. Resectio auris int. total. wesentlich mit der Fraise Eröffnung des Meatus acust int; aus der Öffnung strömt Zerebrospinalliüssigm keit in reichlicher Menge hervor. Während des Fraisens einmal Fazialis. zuckungen. Inzision der l) u ra cerebelli vor dem sinus; es strömt blutige Flüssigkeit beraus. I $\mathrm{nz}$ ision der $\mathrm{D}$ ur a ce re bri der granulationsbe. setzten Partie entsprechend, sowie Inzision der davon gedeckten Hirnmasse; kein Eiter. Punktion des sinns sigmoid. ohne Blut. Inzision des Sinus deckt ein ganz frisches, dunkelrotes Koagulum auf. Resektion der àuberen Sinuswand, wobei Blutung vom oberen Knie, aber nicht von dem horizontalen Teil des Sínus sigmoid. her. - Mikrosko pie der Thrombe. In Bouillon Reinkultur von Streptokokken. Sogleich nach dem Erwachen aus der Narkose mittelstarke, periphere linksseitige Fazialisparese. Spontaner Nystagmus unverandert.

5. Jan. Temp. 38.8-39.7-39-38.5. Puls 120-128-112-108. Reichjiches Aussickern von Zerebrospinalflüssigkeit. Kernig zugenommen.

6. A p r i 1 Temp. $38.7-39.6-40.7-40.1$. Puls $103-100-120-128$. Oph thalmoskopie o. B.

7. April Temp. 40.3-40-40.1-39.1. Puls 128-120-128-114. Noch immer kein Erbrechen oder Schüttelfrost. Etwas benommen und unklar. Unreinlich. Nystagmus zweifellos abgenommen, jetzt hauptsăchlich horizontal nach rechts und nur beim Blick nach rechts vorbanden. Kernig sehr stark. Keine Nackensteifigkeit. Lumbalpunktion entleert $10 \mathrm{ccm}$ milchig trüber Flüssigkeit. Mikroskopie: Reichliche Menge weißer Blutkörperchen. (Lymphozyten: Leukozyten $=1: 3$ ). Keine Mikroben. Kein Wachstum auf Agar oder in Bouillon nach 72 stunden.

10. April Temp. 40-40.2-40-39.6. Puls 120-120-124-120. Zustand unverändert. Heute nur ganz schwacher spontaner Nystagmus, derselben Art wie am 7 . April. Fazialisparese ganz leicht. Kernig noch etwas zugenommen. Keine Nackensteifigkeit.

12. A p ril Temp. 40-40.2-39.8-39.7. Puls 128-124-130-124. BewuBtlos. Starb um 3 Uhr nachmittags.

Sekt i o n. Starke Kontusion des Hirngewebes rings der Inzision, aber kein AbszeB. 
Epikrise: Bei der Aufnahme war die diagnostische Aufgabe vorerst die, zu entscheiden, welcher Ursache das vorhergehende Erbrechen und der noch vorhandene Sohwindel zuzuschreiben sei. Am nächsten lag natürlicb der Verdacht einer Labyrinthitis, aber die Untersuchung bestätigte keineswegs diesen Verdacht. Pat. hatte zweifellos Gehör an beiden Ohren. Es war kein spontaner Nystagmus da, auch nicht während eines von uns beobachteten Schwindelanfalls. Bei der Fistelprobe gab Pat. zwar an, daß sie bei der Kompression Schwindel und Druckempfindung verspurte, aber es war kein Nystagmus zu beobachten, und die Kompression brachte beiderseits identische Drehempfindungen hervor. Nach diesem Ausfall der Fistelprobe lehnten wir die Diagnose eines Labyrinthkapseldefektes ab; sonst hätten wir ja Defekt an beiden Seiten diagnostizieren müssen und zwar an versehiedenen Stellen. Der Vestibularapparat war beiderseits kalorisch sebr leicht reizbar. Demnach war eine Labyrinthdestruktion mit Sicherheit auszuschließen. Ob ein Leiden des Labyrinthes nicht destruktiver Art vorlag, war nicht festzustellen, und besonders nicht an welcher Seite, da die Gleichgewichtsstörungen taberhaupt nicht stark ausgesprochen waren, und außerdem zu vieldeutig sind, um diagnostisch verwertet zu werden. Zeichen einer intrakraniellen Komplikation waren nicht vorhanden.

Wir entschieden uns, die Totalaufmeißelung links vorzunehmen, da hier die Polypenbildung uns eine akute Verschlimmerung der chronischen Eiterung anzudeuten schien. Bei der Operation wurde außer einem osteitisehen Herd an der Medialwand des Antrum nichts Verdåchtiges gefunden. Nach dem Erwachen aus der Narkose Wohlbefinden, bis eine plötzliche Verschlimmerung des Zustandes etwa 15 Stunden nach der Operation einsetzte, indem Pat. vom Erbrechen und Schwindel ergriffen wurde. Der jetzt zugekommene starke spontane Nystagmus sowie der exquisite Drehschwindel sehienen die akute Labyrinthzerstörung außer allen Zweifel zu stellen, und der Ausfall der Hör- und Vestibularisprüfung bestätigte diese Diagnose vollauf, wobei die Unzuverlässigkeit des Weberschen Versuchs klar hervortrat. Jetzt galt es festzustellen, ob schon eine Leptomeningitis hinzugetreten war, wie es das Ansteigen der Temperatur und der ganz leichte Kernig befürchten ließen. Die 24 Stunden naeb der ersten Operation vorgenommene Lumbalpunktion ergab mikrobenfreie, aber milohig getrübte, an 
Lymphozyten und besonders an polynukleären Leukozyten reiche Zerebrospinalflissigkeit. Damit stand die Diagnose Leptomeningitis für uns fest, und unser therapentisches Vorgehen war uns klar vorgezeichnet. Leider vermochte die sofort ausgefuhlte Labyrinthresektion und Öffnung des Subduralraumes in der Fossa cranii med. et post. nicht den tödlichen Verlauf der Meningitis zu ändern.

Die Frage, die uns in diesem Falle am meisten beschäftigt hat, und die wir selbst jetzt nicht mit Sicherheit zu beantworten wissen, ist die nach der Ursache der postoperativen LabyrinthitisDie verdäehtige Stelle an der medialen Antrumwand, die bei der ersten Operation nur mit Vorsicht ansgelöffelt wurde, eben um nicht eine Labyrinthitis hervorzurufen, schien bei der gründlichen Untersuchung gelegentlich der zweiten Operation nicht mit dem Labyrinth in Verbindung zu stehen. Natirlich kann dennoch eine nicht makroskopische Verbindung bestanden haben und wir haben die Infektion durch unsern ersten Fingriff verschuldet Oder es kann sich um eine der nicht seltenen postoperativen Labyrinthinfektionen ohne gröberen Durchbruch der Labyrinthkapsel gehandelt haben. Wie dem auch sei, die Infektion hat hier kaum ein völlig gesundes Labyrinth vernichtet, war ja doch schon vor der Aufnahme Erbrechen und Schwindel aufgetreten. Diese Vermutung unsererseits, daB das Labyrinth schon vor der ersten Operation leidend (aber gewiß nicht vernichtet) war, haben wir durch die Stellung der klinischen Diagnose Otitis interna serosa ausdrücken wollen, ohne damit etwas Näheres über die Beschaffenheit des vermuteten Leidens aussagen zu wollen.

Bei der Diskussion eines anderen Falles (siehe S. 48) haben wir der Bestrebungen verschiedener Autoren erwähnt, die darauf ausgehen, die dureh mikrobenfreie trtibe Zerebrospinalflissigkeit charakterisierten Fälle von Meningitis als nicht zur Meningitis purulenta diffusa gehörend anerkennen zu wollen. Wir baben da die Aufmerksamkeit darauf hingeleitet, dab das Lumbalpunktat selbstredend nicht $\mathrm{n}$ a $\mathrm{ch}$ w e is bare Mikroben $\mathrm{zn}$ enthalten braucht, obgleich eine bakterielle Infektion der Subduralräume schon stattgefunden hat. Wir glauben in diesem Fall einen Bew eis fur die Richtigk eit dieser Ansehaung z zu besitzen. In diesem Fall sind meningitische Symptome schon 24 Stunden nach der das als einen Teil des Subarachnoidalraumes zu betrachtende Labyrinthinuere infizierenden Operation vorhanden. 
Die trtibe Zerebrospinalflussigkeit ist zu dieser Zeit mikrobenfrei, wie auch bei der zweiten, 72 Stunden später vorgenommenen Lumbalpunktion, das heißt zu einer Zeit, da die meningitischen Symptome sehr stark ausgesprochen sind. Es scheint uns, daB es eine sehr gesuchte Erklärung wäre, falls man hier annehmen wollte, dab die Bakterien wälrend der ersten 96 Stunden der Infektion im Labyrinthe verblieben waren, durch ihre Toxine aber die Auswanderung von Lymphozyten und polynukleäre Leukozyten sowie die tibrigen meningitischen Symptome verursacht hatten.

Der Fund bei der zweiten Operation von einem epiduralen Abszeß, einer Pachymeningitis externa und einer Sinus- und Bulbusthrombose war ja gewiB interessant und mußte unser therapeutischesHandeln beinflussen, aber da diese Komplikationen weder in der Anamnese, noeh im späteren, ganz von der labyrinthogenen Meningitis beherrsehten Verlauf der Krankheit Spuren gesetzt haben, stehen wir vor einer weiteren Diskussion ab. Nur möchten wir feststellen, daß der Pachymeningitis externa entsprechend keine Pachymeningitis interna oder Gehirnabszeb gefunden wurde.

6. Sotie A., (No. 172, 1909) 67 Jahre. aufgen. 5. Juni 1909, gestorben 15. Juni 1909.

K linische Diagnose: Otit. med. acut. supp. sin. - Osteit. acut. proc. mast. sin. - Leptomeningitis diffusa purnlenta p. op.? - Kyphoscoliosis.

Sektion verboten.

Anamnese: Vor 10 Tagen Ohrschmerzen links, mit Parazentese behandelt. - Spärliche Eiterung. Gestern Vruckemptindlichkeit hinter dem linken Ohr. Einmal Erbrechen.

Status praesens: Aur. dext.: Trfell atrophisch. Aur. sin.: Im Gehörgang eingedicktes Sekret. Trfell. hervorgewölbt, gerōtet. Para z e n tes e entleert nur Blut. Reg. mast, sin. Etwas Druckempfindlichkeit, bes. über der Spitze. Funk tionsprüfung: Weber nach links. Gebôr făr Flüsterstimme r. $4 \mathrm{~m}, \mathrm{l}$. ad aurem, für Konversationssprache $\mathrm{l}$. $1 \mathrm{~m}$, mit Báránys Lărmapparat im rechten $0 \mathrm{hr}$ ad aurem. Kein spontaner Nystagmus. Kalorische Vestibularisreaktion beiderseits erhalten. Stetho skopie o. B.

Da die Ohrschmerzen an Stärke zunahmen, trotz $\mathrm{Saugbehandlung,}$ entschied man sich für Operation.

12. Juni Temp. 38.2-37.7. Äthernarkose. Resektion des linken Warzenfortsatzes. In den Zellen des Processus Eiter und in der Tiefe eine heftige, ausgebreitete akute Osteitis mit Granulationsbildung und Schwellung der Schleimhant in den Zellen. Wâhrend des Auslöffelns wird Dura und Sinus ein wenig entblößt, scheinen gesund.

13. Temp. 39.8-39.8. Puls 84-64. Ca. 10 Stunden nach der Operation Kältegefühl und Erbrechen, das sich mehrmals später wiederholt hat. Bei der Visite heute schlechtes Aussehen. Keine Nackensteifigkeit oder Kernig. Lumbalpunktion bleibt ,trocken" (Orientierung schwierig wegen Kyphoskoliose). Ophthalm oskopie o. B.

14. Juni Temp. 40.2-40.5-40.6-39.6. Puls 80-64-54-80. Ein mal Erbrechen. Kopfweh in der linken Kopfhälfte und Schmerzen im linken Ohr. Unreinlich. Nackensteifigkeit zweifelhaft, Andeutung von Kernig. 
Kein Nystagmus. Kalorischer Vestibularisreflex erbalten. A thernarkose. Kraniotomie mit Aufdeckung der Dura fossae med. und des Sinus in grober Ausdehnung. Punktion mit Aspiration des Sinus gibt flüssiges Blut. Mikroskopie: Keine Mikroben im Ansstrichprăparat. Kein Wachstum auf Agar oder in Bouillon nach 72 Stunden. $\mathrm{Kr}$ e u z c hnitt d urch die I) u ra. Jodoformgazetamponade zwischen Gehirn und Dura.

15. Juni Temp. 39.5-40.2-40.4. Puls 88-112-68. Nach der Operation kehrte das Bewußtsein nicht wieder. Starb um 1. Uhr vormittags.

E pikrise: 10 Stunden nach der am 12. Juni ausgefuhrten Resektion ad modum Schwartze traten die ersten Zeichen einer ernsten intrakraniellen Komplikation ein. Das auftretende Kältegefuhl konnte den Gedanken auf die Mögliebkeit einer Sinusthrombose hinleiten, aber nach dem Ergebnis der Sinuspunktion am 14 Juni kann diese Diagnose wohl mit ziemlieher Sicherheit ansgeschlossen werden. Danach bleibt nur übrig die Diagnose Leptomeningitis, und zwar postoperative, da die Symptome in direktem Ansehluß an die Operation ersehienen. Die Pathogenese ist hier völlig unklar. Eine Labyrinthitis scheint ausgeschlossen werden zu können, und die operative Entblößung der Dura am 12. als Ursache zu betrachten, kommt uns auch nicht als naturlich vor, da die betreffende Durastelle bei der genauesten Inspektion anläßlich der zweiten Operation durchans intakt zu sein schien.

Sollten wir eine Vermutung aussprechen, wtirden wir an eine „Aktivierung." einer bisher latenten Infektion der Meningen zu denken geneigt sein; für die Annahme einer solehen latenten Infektion könnte vielleicht das einmalige Erbrechen vor der Aufnahme sprecben.

7. Karl Siegfred N., (No. 195, 1909), 6 Jahre, aufgenommen 5 Juli 1909 , gestorben 13 . Juli 1909 .

Klin is ch e Diagnose: Otit. med, chron. supp. dext. cholesteat. - Abscessus subperiostal. proc. mast. dext. - Osteit. acuta in chron. proc. mast. dext. - Abscessus perisinuosus dext. - Epiphlebitis et Thrombophlebitis sinus sigmoid. dext. - Pyaemia. - Leptomeninigitis purulenta diffusa?

Sektionsdiagnose: Thrombosis purulenta sinus petrosi inf dext. Otit. med. Sequelae. - Infarctus purulenti pulmonum. - Degeneratio parenchymatosa organornm.

Anamnese: Seit Jahren Ausflul rechts, jedoch kein Ausfluß während des letzten Jahres. Seit 14 Tagen Ohrschmerzen rechts. Seit zwei Tagen Temperatursteigerung, gestern $40^{\circ}$, und zwei Schüttelfröste. Kein Erbrechen. Gestern einmal Zähnknirschen, kein Schreien.

Status praesens: A ur. dext: Kein Sekret. In der Tiefe des Gebörganges ein Polyp. Reg. mast. dext. Schwellung und oberfiachliche Fluktuation. Keine Schwellung oder Druckemptindlichkeit die V. jugul int dext. entlang. - Aur sin.: 0. B. - Kein spontaner Nystagmus. Kalorische Vestibularisprüfung rechts positiv. - 0 phthalmoskopie o. B. - Kein Kernig oder Nackensteifigkeit. Temp. 37.9.

Zwei Stunden nach der Aufnahme Äthernarkose. Lambalpunkti on entleert $15 \mathrm{ccm}$ fast völlig klare Flüssigkeit. Mikroskopie: Erytbrozyten, vereinzelte Lymphozyten; kein Wachstum auf Agar oder in 
Bouillon nach 48 Stunden. Totala fmeiß elung rechts. Aus dem subperiostalen $A b s z e B$ entleert sich stinkender, grünlicher Eiter, der zahlreiche verschiedene Mikroben, Kokken und Stäbe enthält. Der Fovea entsprechend ist die Corticalis von Granulationen durchbrochen. Die Offnung führt zu einer mit Cholesteatommassen gefüllten Höhle im Warzenfortsatze, die ins Antrum übergeht und nach hinten von der entblößten Sinuswand begrenzt wird. Kraniotomie mit Aufdeckung des Sinus sigmoid. in dessen ganzen senkrechten Verlauf. Die Sinuswand ist oben mit dicken, rötlichen Granulationen besetzt, unten gelbgrün verfärbt und hier findet sich ein kleiner, perisinuöser Abszeß mit, pulsierendem Eiter. Punktion des Sinus gibt kein Blut. Resektion der auberen sinuswand und Entfernung eines Thrombus. Starke Blutung vom oberen Knie her. Rp. Hexamethylentetramin. Mikroskopie des Thrombus: Erythrozyten, Fibrin, reichliche Menge polynukleäre Leukozyten. Keine Mikroben.

5. Juli Temp. 33.7-38.5-40.5-39.4. Puls 120-142-156-118. Heute morgen etwas zyanotisch mit Rigidität der rechten Ober- und Unterextremität, eine Viertelstunde dauernd. Kein Nystagmus. Kernig deutlicb.

6. Juli Temp. 39.6-38.3-41.6-39. Puls 124-104-166-152. Gestern abend Schüttelfrost. Keine Nackensteifigkeit. Kernig bei ca. $120^{\circ}$.

7. Ju li Temp. $39.7-40-40.2-40.8$. Puls 156-142-162-170. Vierwal Erbrechen.

8. Juli Temp. 39.4-39.8-41-41.2. Puls 152-132-160-164. Zweimal heftiges Erbrechen. Sonst Befinden ganz gut. Ist klar. Keine meningitischen Phănomene. Keine Nackensteifigkeit, Kernig sehr zweifelhaft.

9. Juli Temp. 39.4-38.4-40.2-37.5. Puls 168-152-128-140. Gestern Verbandwechsel: Starke Blutung rom oberen Knie her; neue Jodoformgazetamponade, zwei Schuttelfrōste, danach etwas kollabiert aber nach $\mathrm{K} \circ \mathrm{chs}$ alzi $\mathrm{n}$ u si on gebessert. Deutliche Nackensteifigkeit and Kernig.

10. Juli Temp. 40.5-40.4-40.-40.4. Puls 200-170-164-170. Zwei Schüttelfröste, unstillbares Frbrechen, sehr heruntergekommen. Nackensteifigkeit und Kernig wie gestern, sonst keinem eningitischen Erscheinungen. 11. J u li Temp. 39.2-39.6-39.2-40.6. Puls $160-168-144-120$. Schuttelfrost und Erbrechen.

12. Ju $1 \mathrm{i}$ Temp. 40.1-41.5-40.7-40.9. Puls 160-184-188-180. Gestern Schüttelfrost mit Temperatursteigerung auf $42^{\circ}$. Deutliche Nackensteifigkeit.

13. J u li Temp. 40.7-41.4-40.3-41. Puls 160-156-174-200. Nachts Scbüttelfrost. Starb um $7^{3 / 4}$ rormittags.

Bei der Sektion wurde im rechten Sinus transversus ein ziemlich fester, weißer, bis zum Torcular reichender Thrombus gefunden. Im Sinus petros. inf, ein purulenter Thrombus.

Epikrise: Ein gutes Beispiel der beimttickischen Entwickelung einer lethalen intakraniellen Komplikation. Während des letzten Jabres hatten die Eltern keinen Ausfluß bemerkt und Ohrschmerzen nur in den letzten 14 Tagen, Schüttelfrost und Fieber in den letzten 2 Tagen vor der Aufnabme. Trotzdem bereits 2 Stunden nach der Aufuahme der Thrombus im Sinus sigmoid. entfernt wird, gelingt es nicht, das Kind zu retten. Die Todesursache scheinen die bei der Sektion gefundenen purulenten Lungeninfarkte gewesen zu sein. Als die Infektionsquelle muß die purulente Thrombose des Sinus petros. inf. betrachtet werden; wahrseheinlich ist sie von einer primären Bulbusthrombose ansgegangen (keine Blutung vom Bulbus her bei der Sinusresektion). Jedenfalls scheint der Thrombus im 
senkrechten Teil des Sinus sigmoid. nicht infektiös gewesen zu sein (keine Mikroben nachgewiesen); der Thrombus im Sinus transversus ist nachweisbar erst nach dem ersten Verbandwechsel am 8. Juli, 5 Tagen vor dem Tod, gebildet worden, zeigte anch bei der Sektion keine Einschmelzungserscheinungen.

Die mehrmals während des späteren Krankheitsverlanfes festgestellte Nackensteifigkeit und der unzweifelhafte Kernig haben, mit der andauernd hohen Temperatur während der letzten Lebensw oche zusammengenommen, unseren Verdacht auf Le p tomeningitis erweckt. Zwar war die bei der Aufnahme gewonnene Zerebrospinalflüssigkeit norma], aber die Sinusthrombose war natlirlich sehr wohl imstande eine Meningitis zu induzieren, wofür unser nächster Fall einen Beweis liefert. Bei der Sektion wurde für die andauernde Höhe der Temperatur in den Lungenveränderungen eine hinreichende Erklärung gefunden. Ob aber dennoch trotz des negativen Sektionsfundes nicht zu gleicher Zeit eine beginnende Meningitis bestanden hat, läßt sich nicht bestimmt sagen.

8. Anny Marie Elise A. (No. 192, 1909), 12 Jahre, aufgenommen 26. Juni 1900 , gestorben 8 . Juli 1909 .

Klinische Diagnose: Otit. med. acut. supp. dext. - Abscessus perisinuosus dext. - Fpi - et Thrombophlebitis sin. sigmoid. dext. - Leptomeningitis purulenta diffusa. - Albuminuria.

Sektionsdiagnose: Meningitis purulenta. - Thrombosis sinus sigmoidei. Otitis med. dext. - Cystitis. - Degeneratio parenchymatosa organorum.

Anamnese: Seit 2 Jahren zeitweise Ausfluß rechts. Niemals vorher Schmerzen. Seit 2 Tagen Ohrschmerzen rechts, Druckempfindlichkeit des rechten Warzenfortsatzes sowie etwas Drehschwindel in aufrechter Stellung. Kein Erbrechen.

Status praesens. Febriles Aussehen, wohlgenährt. Aur. dext.: Reichlicher mit Epithel vermischter Eiter ohne Geruch. Trfell rot, nur wenig geschwollen, Manubrium deutlich zu sehen. Vor dem oberen Teile des Manubrium eine kleine Perf. mit pulsierendem Eiter. Dilatation der Perforation. Reg. mast. dext.: Leichte Infektion und starker Druckschmerz, besonders über der spitze. Etwas Druckempfindlichkeit die V. jugul. int. dext. entlang, vielleicht durch geschwollene Lymphdrüsen verursacht. A ur, sin. : o. B. Funktionsprü fung: Gehör für Flüsterstimme rechts $10 \mathrm{~cm}$, mit Báránys Lärmapparat im linken $\mathrm{Obr}$ ad aurem. Leichter spontanen rotatorischer $\mathrm{Ny}$ s tagmus nach rechts beim Blick nach rechts, kein Nystagmus beim Blick nach links. Kalorischer Vestibularisreflex rechts vorbanden, tritt etwas spăt ein. Harn obne Album. Kp. Hexamethylentetramin. Stethoskopie o. B.

27. Juni Temp. 39-40.2. Puls 100-102. Recht gut geschlafen. Kein Schütelfrost. Klagt über Stirnkopfweh und Schwindel auch beim Liegen; man kann nichts betreffs Drehempfindung crmitteln. Sie ist uberhaupt etwas trăge. Keine meningitíschen Phänomene. - Heute kein spontaner $\mathrm{Ny}$ stagmus. Um 11 Uhr vormittags $\ddot{A}$ thernarkose: Lumbalpunktion entleert $20 \mathrm{ccm}$ völlig klare Flüssigkeit. Milkroskopie: Keine zellulären Bestandteile; kein Wachstum auf Agar nach 48 Stunden. Resektion des Warzenfortsatzes rechts. Beim ersten Meibelschlag strömt unter starkem Druck Eiter (Mikroskopie: Streptoc. pyog.) hervor aus einer Höhle, 
deren Hinterwand von dem weit nach vorne gelagerten blobliegenden Sinus und deren Vorderwand vom Knochen im Sulcus sigmoideus gebildet wird. Es finden sich keine Zeichen akuter Osteitis. Im Antrum nur geschwollene Schleimhaut. Die Sinuswand mit spinngewebeartigem Belag versehen. Punktion des Sinus gibt kein Blut. Kraniotomie mit Abdeckung des ganzen senkrechten Teils des Sinus sigmoid. und Resektion der $\ddot{a} u$ Beren Sin uswand nebst Entfernung eines ganz rezenten Thrombus von roter Farbe. Blutung aus beiden Enden des Sinus. Mikrosko p i e des Sinusthrombus: Fibrin, Erythrozyten nebst reichlichen polynukleären Leukozyten. Haufen von Kokken, darunter Diplokokken.

28. J uni Temp. 40.1-40.3-39.7-38.5. Puls 104-96-102-90. Kein Erbrechen nach der Narkose, erst heute morgen zweimal. Keine menigitischen Phänomene.

29. Juni T e mp. 38.1-41.1-40-39. Puls 96-120-116-94. Gestern abend schien sie sehr leidend, war etwas zyanotisch, verneinte aber Kälte zu verspüren; auch war kein Schüttelfrost oder Schweiß da. Einmal Erbrechen. Kein spontaner Nystagmus. Kaum Nackensteifigkeit, aber wie es scheint, beginnender Kernig. Starker Druckschmerz der V. jugul. int. entlang (Lymphdrüsen, Vene). Ligatura V. jugul. int. et facialis dext. Die Vene war nicht thrombosiert.

1. Juli Temp. 39.8-39.8-38.6-39.4. Puls 100-88-82-80. Kein Erbrechen oder Kälte. Klagt nur über Stirnkopfweh. Ist lebhaft und natürlich. Kaum Nackensteifigkeit, aber leichter Kernig beiderseits.

3. Juli Temp. 39.6-39.9-39.8-39.2 Puls 112-104-102-100. Gestern nach der Visite ănderte sich plötzlich der Zustand der Pat., indem sie nicht mehr auf Fragen antwortete und mit leerem Blick vor sich hinstierte. Außerdem etwas mangelhafte Aktion des rechten $\mathrm{N}$. facialis und leichte Kontraktur der Flexoren der rechten oberen Extremität. Kernig deutlich beiderseits, Babinski rechts. Schnell trat Sopor hinzu, weshalb gestern $\mathrm{Nm}$. Lumbalpunktion (,trocken") und Kraniotomie mit Abdeckung der Dura, dem Tegmen tympani et antri entsprechend. Dura, die etwas gespannt und byperämisch war, wurde inzidiert. Inzisionen im Gehirn gaben keinen Eiter.

Der soporöse Zustand hielt sich dauernd wăhrend der letzten Tagen ihres Lebens. Nur ab und zu reagiert sie auf Zurede. Am 4. Juli starkes Zurückziehen des Kopfes. Lähmung des rechten Fazialis, besonders dessen Mundast und am 5. Juli der Ober-, am 7. der Unterextremität rechts. Das Fieber schwankte $\mathrm{zwischen} 38.2$ und 40.1. Am 8. Juli $9^{3 / 4}$ Uhr vorm. trat der 'Tod ein.

Bei der Sekti on wurde eine Thrombose des Sinus sigmoid. in der Nähe der Resektionöffnung gefunden, aber nicht im Bulbus oder den übrigen Sinus. Im Meningitiseiter Pneumokokken.

Epikrise: Die am meisten auffallende Eigentümlichkeit dieses Falles ist durch den Umstand gegeben, dab sich bei der Operation keine die Sinusthrombose vermittelnde Osteitis nachweisen ließ. Der pathologische Prozeß war so weit zur Ruhe gekommen, daf sich im Proc. mast. - trotz der ausgesprochenen Druckempfindlichkeit - nichts makroskopiseh Abnormes nachweisen ließ, wäbrend dieSchleimhant imAntrumnoch immer deutlich geschwollen war. Dennoch fand sich ein ziemlich beträchtlicher perisinuöser Abszeß und eine Sinusthrombose mit Kokken und Diplokokken im Thrombus. Nach der Ausräumung des Thrombus trat aber die gehoffte Besserung nicht ein. Es traten zwar keine Schüttelfröste auf - die zwei Tage später ausgefluhrte Unterbindung der V. jugul. int. war durch verdächtige Tem- 
peraturschwankungeu und starke Druckempfindlichkeit in der Venengegend indiziert - aber es stellte sich ein leichter Kernig ein, und vier Tage nach der Operation manifestierte sich die Meninigitis in unzweifelhafter Weise.

Es liegt am näcbsten, den Ursprung dieser Meningitis auf die nachgewiesene Sinusthrombose zu beziehen. Eine Labyrinthitis als vermittelndes Glied läßt sich mit ziemlicher Sicherheit ausschließen, da das betreffende Labyrinth vor der ersten Operation funktionierte, und nach der Operation kein Nystagmus eintrat; doch fehlt eine Labyrinthuntersuchung nach der Operation. Bei der plötzlich eintretenden manifesten Erscheinung: der Meningitis wurde Kraniotomie und Inzisionen des Temporallappens vorgenommen, da jie Möglichkeit ja nicht anszuschließen war, daß es sich um eine durch die Perforation eines Gehirnabszesses durch eine oder subdurale Eiteransammlung hervorgerufene Meningitis handeln könne.

9. Agnes N., (No. 27, 1909). 11/2 Jahr. Aufgenommen 2. Januar 1909 Gestorben 2\%. Januar 1909 .

Klinische Diagnose: Otit. med. chron. supp. cholesteat. sin. Osteitis acuta in proc. mast. sin. - Otitis interna diffusa chron. supp. sin. Bronehopneumonia. - Leptomeningitis suppurat. difusa.

S e k t i o n s d a g n o s e: Meningitis purul. circumscripta et diffusa.

A n a $m$ n se: Seit einem Jahre Ohrflul links, seit einer Woche Schiefheit des Gesichts. Kein Erbrechen oder Schwindel.

$\mathrm{St}$ a $\mathrm{t}$ s p $\mathrm{r}$ a sens: Totale periphere Facialisparalyse links. A u r. d ext $\quad 0$. B. - A u r. s in. Im Gehörgang stinkender Eiter. Totaldefekt des Trf. - R eg. mast. sin. Leichte Infiltration. F un $k$ tions prâfung. Kein spontaner Nystagmus. $\mathrm{F}$ i s te $\mathrm{l}$ pro b e negativ beiderseits $\mathrm{Dr}$ e hvers u c b: Nach Rechtsdrehung kein Nachnystagmus, nach Linksdrehung starker, horizontaler nach rechts gerichteter Nystagmus von 20 Sekunden Dauer. Wiederholte Versuche geben dasselbe Resultat. Kalorische Vestibularisprüfung: Beiderseits negativ. Wăhrend der Ausspülnng rechts macht Pat. eine plotaliche heftige Kopfbewegung; bei nachfolgender Otoskopie sieht man eine frische Perforation des Trf.

Drei Stunden nach der Aufnahme in Athernarkose Lumbalpunktion mit Entleerung von $15 \mathrm{ccm}$ klarer Zerebrospinalflüssigkeit. Mikroskopie: Vereinzelte Lymphozyten, keine Mikroben. - Danach Total a $\mathrm{u}$ fmeibelung links. In den Zellen und im Antrum Cholesteatom. Prominentia can. semicirc. ext. o. B. Die Knochenwand des Can. facialis o berhalb der Fen. ovalis rauh, injiziert. Hinter dem ovalen Fenster findet sich ein halbfester, in Granulationen eingebetteter Strang (freiliegender N. facialis?). Das Fenster ist leer, die Offnung etwas gröber und mit Granulationen gefült, als normal, der Rand raub. Die Sonde dringt in eine weiche im Vestibulum sich befindende Masse ein. Promontorium rauh. Resect. aur. int. totalis. sin. In den Kanälen wurde nichts Abnormes gefunden, nur in der Ampulia can. sem. ext. war die Schleimbaut vielleicht etwas geschwollen. Kein Eiter im Labyrinth. Wăhrend der Narkose wurde bei Manipulationen an den Bogengăngen starker, hauptsächlich horizontaler, Nystagmus nach der operierten (linken) Seite beobachtet.

3. Ja nuar Temp. 38.4-37.8. Nach dem Erwachen aus der Narkose kein Nystagmus, selbst beim Blick nach links. Kein Erbrechen, vōlliges Wohlbefinden. 
4. Jan. Temp. 38.1-38.9. Husten. Stethoskopie: Dämpfung in der rechten Infrascapularis mit bronchöser Resp. ohne Rassein. Ophtbalmosk o pie normal.

5. Jan. Temp. $38.3-39.3$.

6. Jan. Temp. 38.5-38. Hustet weniger. Betinden besser.

Von 14. Jan. ab normale Temp.

21. Jan. Temp. 37.6-36.8. Äthernarkose. Sekundäre Gebörgangsplastik. Sekund a re $\mathrm{Sutu} \mathbf{r}$ der retroaurikulären Inzision.

22. Jan. Temp. 38.9-39.6. Sehr unruhig seit der Operation gestern. Um 4 Uhr nachmittags klonische Znckungen der rechten Korperhälite und Gesichtshälfte. In den linksseitigen Extremităten keine Zuckungen, aber tonische Streckkontraktur. Konjugierte Deviation nach der operierten Seite. 31 Stunden nach der letzten Operation $L u m b$ al $p u n k t i o n$ mit Entleerung von $10 \mathrm{ccm}$ leicht getrübter Flüssigkeit. Mikrosk o pie: Eỉne ziemliche Menge von Lymphozyten. Vereinzelte polynukleăre Leukozyten und eine reichliche Menge ron Gram-positiven Diplokokken von der Form des Pneumokokken, teilweise mit Kapsel verseben; weniger zahlreich ist ein Gram-negativer. mittelfeiner Stab vorhanden. Verband we chsel: Die Wundränder verklebt, ohne Röte oder Schwellung. Keine Retention. Starb um 6 Uhr nachmittags.

Sektionsberich t (Nur das Kranium wurde geöffnet): Nach Eröffnung des Kranium findet man links eine purulente Meningitis. Der verbältnismäßige spärliche Eiterbelag reicht an der Basis bis zur Medianlinie. Entsprechend dem Tegmen tympani liegt anf der zerebralen Seito der Dura ein größerer Eiterherd; von diesem aus als Mittelpunkt nehmen die meningitischen Veränderungen peripberwärts ganz allmäblich $a b$. Der Eiterherd verbarg eine querverlaufende, lineäre pathologische Verdünnung der Dura, an deren medialem Ende eine Perforation. Die Verdûnnung entsprach der Begrenzung des bei der Resektion des Innenohres gesetzten Defektes des Tegmen tympani.

Epikrise: Was diesem Fall sein besonderes Interesc gibt ist erstens die vor der Operation gestellte Diagnose einer Labyrinthdestruktion und zwar einer seit einiger Zeit bestehenden; zweitens der tödliche Ausgang der Krankheit.

Wenn, wie in diesem Fall, eine periphere Fazialisparalyse sich an eine Ohreiterung schliebt, hat man wohl immer an die Möglichkeit einer Infektion des Labyrinthes gedacht, aber bei kleinen Kindern (unsere. Patientin war nur $1 / / 2 \mathrm{Jahr}$ ) war es wie bekannt vor gar nicht langer Zeit unmöglich diese Möglichkeit zu bestätigen oder abzuweisen. Seit Báránys Arbeiten sind wir entschieden besser gestellt. Zwar habe ich (s. M. f. Ohk. 09, S. 470) nachgewiesen - und das Verhalten des rechten Ohres dieses Falles ist ein Beispiel davon - daß es bei Kindern in den ersten Lebensjahren nicht selten unmöglich ist den kalorisehen Vestibularisreflex bervorzurufen, obgleich die Ohren anseheinend normal sind. Daher läbt sich bei diesen Patienten nur der positive Ausfall der kalorischen Vestibularisprüfung verwerten, und der Mangel von kalorischem Nystagmus links hatte hier nach unserer Anffassung keinen diagnostischen Wert. Selbst wenn die kalorische Prufung des rechten (gesunden) Ohres positiv ausgefallen wäre, würden 
wir den Mangel von kalorischem Reflexe links bei einem Patienten in den ersten Lebensjahren kein Gewicht zugemessen haben. Dagegen war der Ausfall des Drehversuches bei wiederholter Prufung sehr sprechend. Nach Rechtsdrehung keine Spur von Nachnystagmus, während ein starker nach rechts gerichteter horizontaler Nystagmus von etwa 20 Sekunden Dauer nach Linksdrehung anftrat. Dies Resultat zeigte, daß der Mangel von kalorischem Nystagmus rechts nicht einer Destruktion des diesseitigen Vestibularapparates zuzuschreiben war und machte höehst wahrscheinlich, daß der linke Vestibularapparat vernichtet war. Der Operationsbefund sowie der Verlauf nach der Operation bestätigte vollauf diese Annahme; es trat nämlich nach der Resektion des Innenohres keine Andeutung von Schwindel oder Nystagmus auf, was sicher der Fall gewesen wäre, falls der Vestibularapparat noch hätte fungieren können. Daß während der Operation an den Bogengängen - in der Narkose - ein starker, hauptsächlich horizontaler Nystagmus nach der operierten Seite vorhanden war, bedeutet nur, daß die Vestibularisn erven nicht degeneriert waren, weshalb bei ihrer mechanischen Reizung: oder Verletzung vestibuläre Reflexe sich hervorrufen ließen. Auffallend ist nur, daß trotz der Narkose wirklicher Nystagmus und nicht nur langsame Augenbewegungen vorhanden waren.

Nach der Operation machte Pat. eine Pneumonie durch, befand sich aber sonst wohl. Daß alles aber nicht so gut war, wie es schien, zeigte sich mit trauriger Deutlichkeit, als nach einem unbedentenden Eingriff (sekundäre Gehörgangsplastik und retroaurikuläre Sutur) ein perakute Leptomeningitis einsetzte. Die Sektion brachte die Erklärung. Dem stehengebliebenen Rande des Tegmen tympani entsprechend war die Dura lineär verdlinnt und an einer Stelle völlig perforiert. Diese Verdinnung ist wahrscheinlich vom pathologischen Vorgang im linken Ohr angebahnt worden, nud die Dura ist dann nach der erste Operation durchs "Reiten" auf dem Knochenrand so viel in ihrer Ernährung geschädigt worden, daß die Perforation einmal eintreten mufte, selbst wenn kein zweiter Eingriff ausgefuhrt worden wäre.

10. Gottli eb Juli us A., (No. 284, 1909), 57 Jahre, Tischler. Aufgen. 16. Okt. 1909. Gest. 3. Nov. 1909.

$\mathrm{K}$ l i $\mathrm{n}$ is $\mathrm{c}$ h e D i a $\mathrm{n}$ o s e: Otit. med, acnt. supp. sin. - Osteit, acuta proc. mast. sin. - Epiphlebitis sinus sigmoid. sin. - Thrombophlebitis sinus sigmoid. sin. p. op. - Septico pyaemia. - Pneumonia.

Sektionsdiagnose: Otitis media. - Oedema meningum. Thromb ophlebitis purulenta sinus transv. - Hypostasis et Oedema pulmonum.

A n a m n e se: Seit acht Tagen Ohrschmerzen und Ausfluß links. Die letzten zwei Tage Druckschmerz hinterm linken Ohr. Status praesens. 
A u r. s i n. Sehr reichlicher Schleimeiter ohne Geruch. Senkung der hinteren oberen Gehörgangs wand. Im hinteren Teil des Trf. große Perf. mit pulsierendem Eiter. Proc mas t. s in.: Druckschmerz an der Spitze. Keine deutliche Infiltration. - A u r. dext.: Trf. zeigt katarrhalische Verănderungen.

20. O k t o ber. Temp. 37.7-37.2. Schmerzen, Druckschmerz und Sekretion unverändert. Nachmittags: A et hernar kose. Res ect. proc$\mathrm{m}$ a $\mathrm{s}$. si $\mathrm{n}$. Die Schleimhaut der ziemlich zahlreichen Zellen geschwollen. In den Terminalzellen und im Antrum Granulationen. Das Knochengewebe morsch, hyperämisch. In einigen Zellen und im Antrum Eiter. Sinus wird ein wenig aufgedeckt, seine Wand ist kaum ganz normal. Durch Ausgleiten

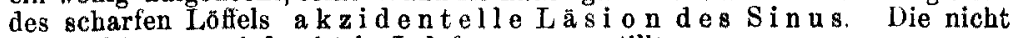
starke Blutung wird mittels Jodoformgaze gestillt.

21. $0 \mathrm{kt}$. Temp. 37.7-37.8. Einmal Erbrechen unmittelbar nach dem Erwachen aus der Narkose. Klagt über Schmerzen in der ganzen linken Hälfte des Kopfes.

22. O k t. Temp. 39.2-38.3. Puls 89-76. Schmerzen zum kranialen Teil der Reg. parotidea, sternocleidomast. und lateralis colli links lokalisiert. Hier Druckschmerz selbst für leichte Berührung. Angina.

23. $0 \mathrm{k}$ t. Temp. 40-40.2. Puls 90-90. Viermal Erbrechen. Heute morgen um 6 Uhr Schüttelfrost, eine Viertelstunde dauernd. Etwas Druckemptindlickeit dem Verlauf der V. jugul, int. sin, entsprechand, aber keine nachweisbare Schwellung.

24. Ok t. Temp. 40.2-39.3. Puls 92-88. Zehn- bis elfmal Erbrechen, siebenmal wässerige Darmentleerungen. Etwas dyspnoisch, nicht zyanotisch. Kein Zeichen von Meningitis oder Febr. typh. Widal negativ. Ophthal: mosk op i e naturlicb. Lumbalpunktion, "trocken".

25. 0 k t. Temp. 39.8-40.4-39.3-39. Puls 85-90-80-72. Zustand wesentlich unverändert. Stethoskopie: Zeichen einer Verdichtung des rechten unteren Lungenlappens. H a $\mathrm{r}$ enthält Albumen.

26. $0 \mathrm{k}$ t. Temp, 39.1-39.5-39-38.2. Puls 72-78-80-72. Gestern Wohlbefinden. Heute morgen um $4 \mathrm{Uhr}$ ein $\mathrm{S} \mathrm{c}$ bu $\mathrm{t}$ telf $\mathrm{r}$ os t mit Temperaturanstieg bis zu $40^{\circ}$. Verband $\mathrm{r}$ ech sel: Der Sinustampon wird obne Blutung entfernt. Sinuswand in der Ausdehnung einer Erbse entblöBt. Die Druckempfindlichkeit im Trigon. carot. stark abgenommen.

27. $0 \mathrm{k} \mathrm{t}$. Temp. 37.5-38.3-39.3-39.9. Puls 72-72-50-80. Gestern abend und heute morgen Erbrechen. Nachts unklar, wollte aufstehen. Bei der Visite benommen. Keine menigitischen Phänomene. A et ber nark os e. K r ani ot om ie. Der ganze senkrechte Teil des Sinus sigmoid. wird aufgedeckt. Die Perforationsöffnung in der Sinuswand hat etwa die Gröbe eines Stecknadelkopfes, ringsumber etwas Eiter. Innerhalb der Perforation ein zerfallender Thrombus. Entfernung desselben nach Inzision der ganzen äußeren senkrechten Sinuswand. Blutung von dem oberen Knie und rom Bulbus her. Mikroskopie des Sinusthrombus: Streptoc. pyogenes. Auf Agar zahlreiche Kolonien des Streptoc. pyog., vereinzelte des Staphyloc. pyog. albus.

25. Okt. Temp. 39.8-39-39.6-38.9. Puls 80-88-72-88. Ziemliches Wohlbefinden, zeitweise doch etwas unklar. Kein Erbrechen.

30. $0 \mathrm{kt}$. Temp. 37.3-39.2-40.4-36. Puls 76-96-104-80. Benommen.

31. Okt. Temp. 38.3-38.5-39.2-39.2. Puls 92-88-92-96. Unreinlich. Bei immer stärker getrübtem Sensorium und unter fortgesetztem pyămischem Fieber starb Pat. am 3. November.

Epikrise: Dieser Todesfall mub leider als durch ein unglückliges Ereignis während der Mastoidaloperation verursacht betrachtet werden, jein Ereignis, das wohl an den meisten viel operierenden Kliniken von Zeit zu Zeit eintrifft, fur gewönlich aber keine ernsten Folgen mit sich fuhrt. Daher waren wir auch nicht besonders unruhig für das Schicksal des Kranken, nachdem wir die bei der akzidentellen Sinusläsion entstehende

Archiv f. Ohrenheilkunde. Ba. 82. 
Blutung mittels Jodoformgazetamponade gestillt hatten. Die 24 Stunden nach der Operation einsetzende Temperatursteigerung möchte der gleichzeitig festgestellten Angina zuzuschreiben sein. 60 Stunden nach der Sinusläsion trat aber ein Schtttelfrost anf, und als außerdem anch Erbreohen, Diarrhoe und etwas Druckempfindlichkeit dem Verlauf der V. jugular. int. entlang auftrat, lag die Befurehtung einer postoperativen Sinusthrombose sehr nale. Die Temperaturen waren jedoch nicht pyämisch, so daß wir auch nach anderen möglichen Ursachen des neuen Krankheitsbildes suchten und eine solche in einer Pneumonie zu finden glaubten. 6 Tage nach der Sinusläsion trat indessen aufs neue Schittelfrost auf, und jetzt hatte die Temperaturkurve auch mehr den pyämischen Charakter angenommen. Am nächsten Tage wurde ein obturierender, zerfallender, von der Perforationsstelle zentral- und peripherwärts sich erstreckender Thrombus aus dem Sinus entfernt, aber trotzdem gelang es jetzt nicht mehr den ron Alkoholmißbranch geschwächten Pat. zu retten. Zwar traten keine Schtuttelfröste mehrauf, aber der Organismus war schon zu durchseucht, um sich wieder zu erholen. Besser wäre wohl die Aussicht gewesen, hätten wir uns früber zur Sinuseröffnung entschließen können. Aber auch in solchem Falle wäre wohl der Erfolg fraglich gewesen, handelte es sich eben um eine anßergewöhnlich virulente Infektion, wie es sowohl das klinische Bild als der Streptokokkenfund im Thrombus zeigte.

11. Ketty Kragh L. (No. 181, 1909), 10 Jabre, aufgenommen 13. Juni 1909, gest. 26. Juni 1909 .

Klinische D iagnose: Otit. med. chron. supp. dext. - Seq. Otit. med. chron. supp. sin. cholesteat. - Osteit. acut. in chron. proc. mast. sin. - Abscessus subperiostal. proc. mast. sin. - Abscessus perisinuosus sin. Thromboplebitls sinus sigmoid. sin. - Pyaemia. - Pachymeningitis ext. et int. fossae cranii med. sin. - Abscessus subduralis reg, centromotor. sin. Encephalitis gangraenosa reg. centromotor. sin. - Leptomeningitis purulenta diffusa sin.?

Sektionsdiagnose: Abscessus epicerebralis sin. - Compressio lobi occipit. sin. - Pachymeningitis int. purul. dext.

Dieser interessante Fall wird ausführlich in einer Arbeit uber otogene Pachymeningitis int. veröffentlicht werden.

12. Ore Ejnar E. (No. 203, 1909) 3 Monate, aufgenommen 20. Juli 1909, gestorben 26. Juli 1909 .

Klin ische Diagnose: Otit. med. acut. supp. dext. et sin. - Osteit. acut. proc. mast. dext. - Bronchopneumoniae variae. - Atrophia. - Leptomenigitis?

Sektionsdiagnose: Focus tubercul. lobi inf. pulm. dext. - Tuberculosis miliaris pulmonum, hepatis, lienis, renum, pericardii. - Tuberculosis gland. bronchial, retromaxill, cervicalinm. - Ulcera tuberculosa intestini (et laryngis?) - Hyperaemia et Oedema meningum - Otit. med. supp. dupl, - Otit. interna circumscripta dext. 
A n a m n e e: Wird abergelegt aus einem anderen Hospital, wo Pat. unter Verdacht von Masern aufgenommen worden war.

Status praesens: Sehr elend und atrophisch. Temp. 33.8. Auf Truncus und Femora ein fleckiges, Morbilli-ähnliches Exanthem. Totale periphere Fazialisparalyse rechts. A u $\mathrm{r}$. d ext.: Sehr reichlicher, stinkender Eiter. Gehörgang etwas verengert. In der Tiefe fühlt die Sonde rauhes Knochengewebe. - A u r. s i n.: Spärliche eitrige Sekretion. Gehörgang verengert. In der Tiefe eine rote, geschwollene Fläche. $R$ eg i o n e s $m$ a s t.: o. B. Bedeutende Lymphdrüsensehwellungen in der linken und besonders rechten Reg. retromaxill., submentalis und lateralis colli. Kein spontaner Nystagmus. $K$ a loris $\mathrm{ch}$ e $\nabla$ es tibularis reaktion tritt rechts sofort ein. Fis tel p r o b e negativ. Deutliche Nackensteifigkeit. Die Extremităten werden in andauernder Flexion gehalten. Nach der Untersuchung kollabiert.

21. J u li Temp. 35.5-37.4. Ein wenig gebessert. Hustet viel. Die Fazialisparalyse rechts vielleicht nicht völlig total.

22. J u I i Temp. 38.6-36. Sehr reichliche Obreiterung rechts. Keine meningitischen Symptome außer starker Nackensteifigkeit; Kernig sehr zweifelhaft. St ethos $\mathrm{k}$ o i e. Feines Rasseln beiderseits, Dämplung rechts. Ohne Narkose Resektion des Warzenfortsatzes rechts. Ausgebreitete akute Osteitis. Mikroskopie: Keine Tuberkulose.

23. Juli Temp. 38.8-38.3. Nach der Operation etwas kollabiert.

24. Juli Temp. 39.6-39. IBt weniger und ist deatlich mehr heruntergekommen. Kopf stark nach hinten gebeugt. Kein Kernig.

25. Ju li Temp. 38.1-37.6. Kalorische (Kaltwasser) Vestibularisreaktion rechts sehr heftig und schnell eintretend.

26. Juli Temp. 39-38.1. Außer Nackensteifigkeit und Erröten des Gesichts keine meningitischen Erscheinungen. Starb um 7 Ubr vormittags.

Sektion des rechten Schläfenbeins: Sowohl in der Cisterna perilymphatica als in den beiden Skalen der Cochlea entzündliche Veränderungen. Dagegen scheinen die Cristae acusticae ampull. sup. et ext. normal zu sein. Der mikroskopische Fund wird später ausführlicher veröffentlicht werden.

Epikrise: Bei der Aufnahme dieses elenden, atrophisehen Kindes zweifelten wir gar nicht daran, daß es rechts eine ausgebreitete akute Osteitis des Warzenfortsatzes darbot. Wir wagten jedoch nicht sofort eine Operation vorzunehmen, da bereits die sohonende Untersuchung einen drohenden Kollaps herbeiführte. Erst zwei Tage später, als die Temperatur etwas gestiegen war, wurde ohne Narkose eine Resektion des Warzenfortsatzes ad mod. Schwartze ausgeführt, aber diese Operation vermochte natürlich nicht bei der ausgedehnten tuberkulösen Durehseuchnng des Kranken sein Leben zu retten.

Zwei Punkte in der Krankengeschichte bieten besonderes Interesse dar, erstens die Frage, ob eine Meningitis vorlag, und zweitens die, ob vielleicht auch eine Erkrankung des Labyrinthes vorhanden war.

Der Gedanke an die Möglichkeit einer Meningitis, und besonders einer tuberkulösen Meningitis, mußte durch die sehr ausgesprochene Nackensteifigkeit erregt werden. Es war zwar kein - oder nur zweifelhafter - Kernig vorhanden, aber ob wir auch diesem Zeichen für die Diagnose einer Meningitis sehr 
große Bedeutung zuschreiben, kann es doch in einzelnen Fällen zweifellos fehlen. Leider haben wir keine Lumbalpunktion ausgefubrt, wodurch vielleicht die Frage hätte beantwortet werden können. Denn dadurch, daß bei der Sektion nur Ödem und Hyperämie der weichen Gehirnhäute gefunden wurde, ist die Möglichkeit einer beginn enden Meningitis (tuberkulöser oder anderer Natur) unseres Erachtens durehaus nicht ausgesehlossen. Um die erwähnte Möglichkeit mit einiger Sicherheit abweisen zu können, miibte man eine sehr gründliche mikroskopische Untersuchungd. Gehirnhäute mit negativemResultat vorgenommen haben.

Die zweite Frage läßt sich bestimmter beantworten, was von grober praktischer Bedeutung ist. Bei kleinen Kindern kann man selbstredend keine Gehörprüfung vornehmen. Um den Zustand des Labyrinthes zu beurteilen, ist man also auf die Untersuchung des Vestibularapparates und besonders des kalorischen Reflexes verwiesen. Wie ich gefunden habe, kann man aber bei kleinen Kindern von dem Ausbleiben des kalorischen Nystagmus nicht auf ein Leiden des Vestibularapparates schließen (8. S. 63 unten). Dieser Fall zeigt jetzt, daß man noch 22 Stunden vor dem Tode sehr starke Vestibularisreaktion hervorzurufen imstande ist, und doch sind verschiedene Abschnitte des betreffenden Labyrinthes von ausge. sprochenen entzundlichen Veränderungen ergriffen, die, wie die mikroskopische Untersuchung zeigt, weiter zurück als die letzten 22 Stunden datieren müssen. Mankann also auchnichtvon vorbandenem Vestibularisreflexe anf Intaktsein des ganzen betreffenden Labyrinthes schlieBen, eine Tatsache fur die tbrigens schon einige melyr weniger tiberzengende Belege in der Literatur vorhanden sind. Damit bübt natirlich die kalorischeVestibularispr ü fung ihregroße und unstreitigeBedeutung: nicht ein, nur ist es gut, ihre Begrenzung in diagnostischer Rücksicht sich klar vor den Augen zu halten. Fùr die Zuverlässigkeit des kalorischen Reflexes, wenn man aus seinem Vorhandensein nicht mehr herauslesen will, als es besagt, bringt der Fall einen guten Beweis. Die Crista acustica ampullae can.semicire. sup., von der aus der Reflex nach der Theorie ausgelöst wurde, bot ja keine nachweisbaren Veränderungen dar, obgleich in der nahen Cisterna perilymphatica deutliche Entzlundungserscheinungen zu beobachten waren.

13. Paul Louis P., (No. 133, 1909) 11/2 Jahre, aufgenommen 22. April 1909, gest. 29. April 1909.

Klinische Diagnose: Pneumonia sin. (tuberculosa?). - Empyemat. pleurae sin Seq. - Otit. med. acut. supp. dext. - Leptomeningitis tuberculosa? 
Sektionsdiagnose: Bronchopneumonia dupl. - Tuberculosis gl. bronchial. - Osteitis purulenta proc. mast. dext. - Otitis med. dext.

Anamnese: Früber in einer Kinderklinik behandelt (Diagnose: Pneumonia crouposa sin., Otit med. supp. acut. dupl.). Wăhrend beinahe $z$ wei Monaten Temperaturschwankungen $z$ wischen $37.5-39^{\circ}$. In den letzten Tagen $39-40^{\circ}$. Wird unterVerdacht von otogenerMeningitis transferiert.

Status praesens: Etwas benommen. Nackensteifigkeit. Vielleicht Andeutung von Kernig. Sonst keine meningitischen Phänomene. Lumbal punktion entleert blutige Hlüssigkeit. Aur. dext. Akute Eiterung. Aur. sin. und Proc. mastoid ei o. B. Stetbosk. Pleurotomicöffnung gat offen, sonst nichts Abnormes. v. Pirquet positiv. Ophthalmosk opie natülich.

Wăbrend der folgenden Tage Temperaturschwankungen zwischen 38.5 und ca. $40^{\circ}$. Starke Nackensteifigkeit, ab und zu Erbrechen, viel Husten, aber kein Kernig oder andere meningitische Pbänomene. Starb am 29. April,

Epikrise: Der Fall ist ein gutes Beispiel der diagnostischen Wichtigkeit der Lumbalpunktion. Weder bei der ersten noch bei zwei späteren Punktionen gelang es uns brauchbare Zerebrospinalfussigkeit zu gewinnen. Wir waren daher darauf verwiesen, aus den übrigen klinischen Symptomen die Diagnose zu stellen. Bei der nachgewiesenen Tuberkulose des Kindes schien es uns nicht unberechtigt das Krankbeitsbild auf eine tuberkulöse Meningitis zu bezichen. Die Sektion belehrte uns eines anderen, aber es war kaum möglich gewesen die diagnostischen Schwierigkeiten obne Untersuchung der Zerebrospinal * flussigkeit zu uberwinden. Von Interesse ist der dureh diesen Fall wieder gebrachte Nachweis des fraglichen diagnostisehen Wertes der Nackensteifigkeit bei kleinen Kindern.

14. Anna P., (No. 157, 1909) 6 Monate, aufgenommen 25. Mai 1909, gest. 29. Mai 1909 .

Klinische Diagnose: Paedatrophia. - Otit. med. acut. supp. dext. - Osteit. acut. proc. mast. dext. - Abscessus subperiostalis reg. mast. dext. incip. - Bronchopneumonia? - Meningitis?

Sektionsdiagnose: Otit. med. dext. - Enteritis chronica. - Intumescentia gland, mesenterial. - Atrophia 1. gr. - Intumescentia lienis 1. gr. Hufeisenniere.

Anamnese: In einer hiesigen Kinderklinik wurde am 19. Mai Fazialisparalyse rechts beobachtet, am 23. Mai Obrfluß rechts.

Status praesens: Atrophisches Kind von elendem Aussehen. Ausgebreitete Impetigo. Totale periphere Fazialisparalyse rechts. Aur. dext. Reichlicher Schleimeiter. Schwellung der Gehörgangswände, besonders hinten oben, verbindert genaue Inspektion. Beim Austupfen wird ein an der Gelenkflăche stark kariöser $A m b O B$ entfernt. Rings um das rechte Ohr große geschwollene Glandelpakete. A ur. sin. Cerumen. Funktionsprufung: Kal orischer Vestibularis reflex beiderseits vorbanden. Keine Nackensteifigkeit oder Kernig. Wenige Stunden nach der Aufnabme Resektion des recbten Warzenfortsatzes obne Narkose. Periost verdickt. Corticalis von Granulationen durchbrocheu. Auslöffelung des Prozesses.

26. Mai Temp. 35.5-37.7. Befinden nach der Operation gut.

27. $\mathrm{M}$ ai Temp. 39-39.4. Stethoskopie normal.

28. $\mathrm{M}$ a i Temp. 39.2-40.1. Keine meningitischen Phänomene.

29. Mai Temp. $38.8-42.2$. Starb um $101 / 2$ Uhr vormittags.

Sektion: Nichts im Gehirn oder in den Lungen.

Epikrise: Dieser Fall gibt zu keinen weitläufgen Erörterungen Anlaß. Die gut indizierte Operation wurde ohne 
Narkose ausgeführt um nicht die Vitalität der elenden, heruntergekommenen Pat. mehr als nötig zu gefährden. Das Ansteigen der Temperatur nach der Operation muBte die Aufmerksamkeit auf die Möglichkeit einer Lungenkomplikation oder einer Meningitis hinleiten. Aber die klinische Untersuchung vermochte nicht das Vorhandensein einer der genamten Komplikationen nachzuweisen. Lumbalpunktion wurde wegen der in diesem Falle großen Infektionsgefahr (ausgebreitete Impetigo) nicht vorgenommen. Auch die Sektion brachte kaum eine Erklärung der postoperativen Temperatursteigerung.

15. Henning Hagerup A., (No. 318, 1909) 2 Jahre, aufgenommen 11. Dez. 1909, gest. 18. Dez. 1909.

Klinische Diagnose: Otit. med acut. supp. dext. et sin. - Osteitis acuta. proc mast. sin. - Leptomeningitis purulenta diffusa (cerebrospin.-epidem.?).

Sektion verboten.

An amnese. Am 6. Dezember begann Fieber, am 7. Erbrechen, am 9 . Dezember fing Pat an zu stieren und zu schielen, am 10. zu schreien. Obrfluß beiderseits seit dem Sommer 1908, jedoch kein Ausfluß bemerkt während des letzten balben Jahres. Wird aus der neurologischen Klinik übergelegt.

Status praesens: Heruntergekommen, wimmert und schielt. Deutliche Nackensteitigkeit, Kernig angedeutet. Aur. dext. : Schleimeiter im Geborgang. Trf. diffus gerótet. Proc. brevis deutlich zu sehen. Keine Perforation. A ur. sin.: Eiter im Gehörgang. Große Perf. des Trf. Reg. mast. dext. o. B. - Reg. mast. sin. Leichte Infiltration aber Fovea. Funktionsprüfung: Kalorischer Vestibularisreflex beiderseits vorbanden. Stethoskopie, Ophthalmoskopie o. B. Temp. 40.5. $\mathrm{Puls} 130$. Lumbalp unktion entleert $10 \mathrm{ccm}$ stark eitrige Flüssigkeit. Mikroskopie: Reichliche Menge polynukleäre Leukozyten nebst zahlreichen Gram-negativen Diplokokken, die an mehreren Stellen des Präparates rücksichtlich ihrer Lagerung und Form Meningokokken ähnlich sind. In einem der Präparate außerdem eine Gruppe schlanker Stab - Bakterien. Auf Agar Wachstum eines Gram-negativen Diplokokkus.

Zwei Stunden nach der Aufnahme in Aethernarkose Resektion des linken Warzenfortsatzes. Integumente etwas geschwollen. Oberfläche und Substanz des Knochens ziemlich natürlich, nur auberhalb des Antrums morscles, zerfallendes Knochengewebe. Kraniotomie. Dura der Fossa med. gespannt, hyperămisch. bildung eines viereckigen Duralappens. Das Gehirn wölbt sich vor; kein Exsudat, kein Liquorabflub. Ringförmige Jodoformgazetamponade zwischen dem Gehirn und dem durabekleideten Rahmen der Kraniotomieöffnung.

12. Dez. Temp. 40.4-39.8. Puls 130-108. Dreimal Erbrechen. Starkes Schielen, Erröten des Gesichts, etwas Zähnefletschen. Bewußtlos.

13. Dez. Temp. 39.4-395. Puls 112-116. Kernig und Babinski nicht stark, aber positiv. A ur. dext. Kein Eiter im Gehörgang. Proc. brevis und Manubrium deutlich. Vorn unten eine trockene Perforation.

In den folgenden Tagen schwankten die Temperaturen zwischen 39.2 und 415. Der Zustand verseblechterte sich immerfort. Am 17. Dezember reichliche Ohreiterung rechts (am nicht operierten Obr). Wiederholte Lumbalpunkturen ergaben dasselbe makro- und mikroskopische Bild wie bei der Aufnahme.

18. Dez. Temp. 39.4-41.5. Puls 106-124. Starb um 2 Uhr nachm.

Epikrise. Die erste Frage, die wir bei der Aufnahme dieses Pat. zu beantworten versuchen mußten, war die, wie weit die schon seit einigen Tagen bestehende Meningitis als otogen 
zu betrachten war. Die Lumbalpunktion ergab eine stark eitrige Zerebrospinalflüssigkeit, die sehr viele polynukleäre Leukozyten und, zum Teil dem Meningokokken ähnliche, Gramnegative Diplokokken enthielt. Dieser Fund erschwerte gewissermaßen die Lösung der Frage; die Diagnose Meningitis cerebrospinalis epidemica wurde uns dabei nahegelegt olne mit Sicherheit festgestellt werden zu können. Wir haben uns jedoch für eine Operation entschieden von der Betrachtung ausgebend, daß man die Aussichten des Kranken beim Operieren verbessern würde, falls die Meningitis otogen war, während zu gleicher Zeit eine Operation kaum die Prognose verschlechtern würde fur den Fall, daß es sich um eine genuine Meningitis cerebrospinalis epidemica handelte.

Danach trat die Frage an uns heran, welches Ohr zu operieren sei, da ja an beiden Eiterung bestand. Wir haben uns für das linke entschieden, weil die Entzündung hier am ältesten und stärksten zu sein schien, und weil hier - wenn auch nur leichte - Veränderungen am Proc. mast. vorhanden waren.

Um zur Diagnose zurückzukehren muissen wir bedauern, daß eine Sektion, die vielleicht die Pathogenese der Meningitis hätte klären können, nicht erlaubt wurde. Während des Lebens des Pat. war es den Bakteriologen unmöglich mit Bestimmtheit zu sagen, ob die vorhandenen Mikrooganismen wahre Meningokokken waren oder nicht. Hätten wir fruhzeitig genug die Meningokokkennatur der Mikroben feststellen können, wlüden wir wahrscheinlich nicht operiert haben. Dab wir damit die rechte Wahl würden getroffen haben, ist indessen nicht unstreitig, denn eine "genuine" Zerebrospinalmeningitis mag wohl gelegentlich ebensowohl von einer durch die spezifischen Erreger hervorgerufenen Ohreiterung ansgehen wie von dem gewöhnlich beschuldeten Leiden des Nasenrachens.

16. Ingeborg R., (No. 297, 1909), 1 Jahr, aufgenommen 17. November 1909, gestorben 19. November 1909.

Klin ische Diagn os e: Otit. med. acut. supp. sin. - Leptomeningitis tuberculosa.

Sektionsdiagnose: Otit. med. sin. - Tuberculosis solitar. cerebelli. Meningitis tuberculosa.

An amnese: Vater leidet an Tuberkulose. Vor zwei Wochen. Bronchitis. Vor einem Monat begann Ohrflub links. Vor acht Tagen plötzliche Erkrankung mit Erbrechen und Fieber; gleichzeitig hörte das Ohr auf zu sezernieren. Sie wurde sehr unruhig, schrie fortwăhrend, reichte nach dem Kopfe, schielte. Zuckungen in den Extremitäten. Während der letzten zwei Tage bewabtlos. Puls 150 .

Status praesens: Komatöses, wohlgenăhrtes Kind. Temp. 37.2. 
A ur. sin.: In der Tiefe des Gehörganges etwas Eiter obne Geruch Trommelfell oben rot, hervorgetrieben. Keine Perforation. Kalorische Vestibularisreaktion erhalten. A u r. dext. und Regiones mast. o. B. Starkes Scbielen links. Ausgesprochene Nackensteifigkeit. Kein deutlicher Kernig, aber etwas Rigidität der linken Unterextremität. Leichtes Zittern und kleine Zuckungen in den Armen. Stethosk opie o. B. Lumbalpunktion entleert leicht trübe Zerebrospinalfüssigkeit. Mikroskopie: Ziem lich reichliche Menge mononulieäre Lymphozyten, vereinzelte polynukleäre Lenoszyten, keine Mikroben. Kein Wachstum auf Agar nach 48 Stunden. Inz is ion des linken Trf.

18. Nov. Temp. $37.2-38.8$. Unverändert.

19. Nov. Temp. 40.2. Exitns.

Bei der Se ktion wurde in der linken Zercbellarhemisphăre ein solităres Tuberkel von Erbsengrổe gefunden.

Epikrise: Die Anamnese sowie die objektiven Symptome bei der Aufnahme machten die Diagnose einer Leptomeningitis höohstwahrscheinlich. Fraglich war aber, ob diese Meningitis otogen war, da ja eine Mittelohreiterung vorhanden war. Das Ergebnis der Lumbalpunktion, die in solchen Fällen geradezu unentbehrlich ist, zeigte, das die Meningitis tuberkulösen Ursprungs war, was auch die Sektion später bestätigte. Das solitäre Tuberkel im Zerebellum hatte keine klinischen erkennbaren Erscheinungen hervorgerufen.

17. Otto Henry C., (No. 175, 1909), I Jahr, aufgenommen 10. Juni 1909, gest. 20. Juni 1909.

Klinische Diagnose: Otit. med. acut. supp. sin. - Osteit. acuta proc. mast. sin. - Pneumonia dupl. - Septichaemia.

Sektionsdiagnose: Bronchopneumonia pulmonis utriusque. Nephritis parenchymatosa.

A nam ese: Vor 8 Tagen bemerkte die Mutter, daß der Pat. krank war. Temp. 39 5. Schwellung hinter dem linken Ohr. Vor drei Tagen Ausfuß links. Heute Erbrechen. Während der letzten 2 Tagen mehrmals Schuttelfrost. Keine meningitischen Phänomene.

Status praesens: Ziemlich wohlgenährtes Kind. Etwas Husten. A ur. d ext.: Cerumen. A ur. sin. Reichlicher, stinkender Eiter. Beim Austupfen Blutung. Reg. mast. si n. Etwas diffuse Infiltration, besonders über der Basis. Unterhalb der Spitze des Warzenfortsatzes große Lymphdrüsenscbwellungen. Keine Nackensteifigkeit oder Kernig. Stethoskopie: Leichte Dampfung hinten unten beiderseits mit spärlichem feinem Rasseln.

11. Juni Temp. 38.5-39.9. Keine meningitischen Phänomene. Ophthalmoskopie o. B. Chlor oformnarkose: Lumbalpunktion entleert leicht flockige Flüssigkeit, die mikroskopisch vereinzelte Lymphozyten, aber keine Mikroben enthält, auf Agar kein Wachstum nach 48 Stunden. Resektion des linken. Warzenfortsatzes. Periost stark verdickt. Der akute osteitische Prczeß nicht sehr ausgebreitet. Aufdeckung des Sinus ; Punktion gibt ftüssiges Blut.

12. Juni Temp. 39.6-42.1. Seit der Operation ist der Zustand nicht schlechter geworden.

Die folgenden Tage war die Temp. meistens $40-41^{\circ}$. Das Kind wurde immer elender und hustete viel. Keine meningitischen Symptome. Die Operationkkavităt granulierte gar nicbt, bedeckte sich mit weißlichen Membranen. Pat. starb am 20. Juni um 2 Uhr nachmittags.

Epikrise: Ein ähnlicher Fall wie der folgende insofern die durch ein Lungenleiden hervorgerufene Temperatursteigerung uns beigleichzeitig vorhandener Mittelohrentzîndung an die Möglichkeit 
einer intrakraniellen Komplikation denken ließ. Auch hier wies die Operation nach, daß eine solche (Meningitis oder Sinusthrombose) nicht vorlag, so daß man also vom otiatrischen Standpunkt die Sachlage beurteilend, mit ruhigem Gewissen die unaufhaltlichen Fortschritte der Erkrankung folgen konnte.

18. Jo hanne Andrea J, (Nr. 136, 1909), i Monate, aufgenommen 9. Mai 1969, gest. 10. Mai 1909 .

Klin ische Diagn o se: Otit. med. exsud, sin. - Oedema reg. mast. sin. - Bronchopneumonia dupl. - Morbilli. - Khachitis.

Sektionsdiagn ose: Bronchopneumonia dupl. - Rhachitis l. gr.

An am nese: Seit 26. Februar d. J. in Behandlung in einer Kinderklinik; Diagnose Bronchitis, Impetigo, Enteritis, Morbilli. Seit 12. April eine ausgebreitete impetiginōse Eruption. Am 3. Mai begann das Kind zu fiebern; während der folgenden Zeit Temperaturschwankungen $38.3-40.3$. Am 7. Mai ein masernähnliches Exanthem. Am 8. Mai Temp. 40.3-39.5. Am 9. Mai Temp. 39.5-39.5. Heute Morgen ein Krampfanfall mit universellen Zuckungen von $3 / 4$ Stunde Dauer. Ohrful ist mebrmals links beobachtet worden, auch in den letzten Tagen. Heute deutliche pastöse Infiltration des linken Warzenfortsatzes.

Status praesens: Pat. sehr heruntergekommen, zyanotische Lippen, keuchende und rasselnde Respiration. Puls schlecht. Leichte Rhachitis. Im Capillitium eine ausgebreitetete impetiginöse Affektion mit Abszeßbildung. A ur. sin.: Kerı Eiter im Gebörgang. Trfell rot, geschwollen, hervorgewölbt. Inzision des Trf. entleert seröse Flüssigkeit. Rings um das linke $\mathrm{Ohr}$ ein sebr stark ausgesprochenes Oedem, sich bis in die Temporal- und Wangen region hinein streckend. Keine Fluktuation. Keine Nackensteifigkeit oder Kernig. Lumbalpunktion entleert $t 0 \mathrm{ccm}$ vŏllig klare Flüssigkeit. In Lokalanästhesie Resektion des linken Warzenfortsatzes. Die Weichteile stark infiltriert. Warzenfortsatz sehr pneumatisch. Schleimhaut der Zellen natürlich. Knochengewebe vielleicht ein wenig hyperämisch. Dura und Sinus freigelegt, Ausseben natürlich. Punktion des Sinus entleert normales Blut. Nach der Operation war ein starkes Oedem der rechten Mastoidal- und Warzengegend entstanden. Pat. scheint ein wenig gebessert.

10. Mai Tep. 38.2-41. Nach einer gut verbrachten Nacht starb Pat. ruhig um 10 Uhr vormittags.

Epikrise: Diese Patientin wurde aus einer Kinderklinik zu uns unter der Diagnose otogener Hirnabszeß ubbergelegt. Bestimmend hierfür waren natirlich die vorhergehenden Krämpfen gewesen; doch mußte ihre universelle Ausbreitung eher den Verdacht wach rufen, $\mathrm{dab}$ es sich um genuine Kinderkonvulsionen handelte. Da Pat. fruher eine linksseitige Obreiterung gehabt hatte, und links noch eine exsudative Mittelohrentztindung bestand, muBte man die Mögliebkeit einer intrakraniellen Komplikation zugeben, und fur das Vorhandensein einer eventuellen vermitttelnden Osteitis des Warzenfortsatzes könnte wohl das starke Ödem rings nm das linke Obr sprechen. Wir dachten bei dem hohen Fieber am ehesten an eine Meningitis oder Sinusthrombose. Die Operation aber zeigte, daß der Warzenfortsatz völlig gesund war; noch fand sich etwas Abnormes bei der Lumbal- und Sinuspunktion. 
In diesem Falle haben wir demnach eine falsche Diagnose gestellt und eine unnötige Operation vorgenommen. Doch glauben wir durch die unnötige Operation nicht unsere Pat. geschädigt zu baben, da sie nicht narkotisiert wurde, um nicht eine Versehlimmerung ihres Lungenleidens hervorzurufen, und ilur Zustand nach der Operation entschieden nicht schlechter war als vorher. Endlich meinen wir noeh jetzt, daß eine Operation am Warzenfortsatze durchaus indiziert war, da man bei der obwaltenden Sachlage die Möglichkeit einer intrakraniellen Komplikation nicht ausschließen konnte. Bemerkenswert ist, daß die ödematöse Schwellung der Mastoidalgegend, die den Verdacht einer Osteitis wachrief, sich als die Folge einer Tendenz zur Ödembildung an dekliven Partien ausstelle, indem eine gleichartige ödematöse Schwellung rechts entstanden war, nachdem die Pat, während der Operation mit der rechten Wangenund Mastoidalgegend nach unten geruht hatte.

19. Hans C. (No. 230, 1909), 43 Jahre, Schneider. Aufg. 13. Jali 1909, gestorben 21. Aug. 1909.

Klinische Diagnose: Otit. med. acut. supp. sin. - Absc. subperiost. proc. mast. sin. - Absces. suprasplenicus. - Osteit. acuta p. m. sin. et oss. temp. - Nephritis acuta? chronica? - Uraemia.

Sektionsdiagnose: Degeneratio amyloidea renum, lienis, intestini, gland. supraren, - Seq. resect. proc. mast. - Seq. resect. gen. sin.

Der Fall bietet außer dem ungewöhnlichem Sitz der Osteitisund der Abszesse kein besonderes otologisches Interesse dar. Die amyloide Degeneration war schon bei der Aufnahme nachweisbar und ist vermutlich durch die zur Resektion des Knies führende Krankbeit (Tuberkulose) verursacht gewesen. Doch lälit sich die Möglichkeit nicht von der Hand weisen, daß die mit AbszeBbilaung komplizierte, seit einiger Zeit bestehende Mittelohreiterung von ätiologischer Bedeutung gewesen sei.

20. Svend Aage A. (No. 187, 1909), 2 Jahre, anfgen. 3. Juli 1909, gest. 4. Juli 1909 .

Klin is che Diagn ose: Diphtheritis faucium - Pemphigus. - Catarrh. gastro - intestinal. - Otit. med. supp. acut. dupl. - Sektions diagn ose: Diphteritis faucium, nasi, epiglottidis. - Bronchopneumonia. - Rbachitis 1; gr. - Stasis et steatosis hepatis. - Degeneratio parenchym. renum.

Das klinisebe Bild war hier durehaus von der schweren Rachendiphtherie beherrscht, die auch, ehe der Pat. in ein Epidemiehospital transferiert werden konnte, den Tod herbeifuhrte. 